\title{
Fast Multipole Methods on Graphics Processors
}

\author{
Nail A. Gumerov and Ramani Duraiswami \\ Perceptual Interfaces and Reality Lab. \\ Computer Science and UMIACS \\ University of Maryland, College Park \\ \{gumerov, ramani\}@umiacs. umd.edu \\ Fantalgo, LLC, Elkridge MD \\ info@fantalgo.com ww. fantalgo.com
}

21 Nov 2007, Revised 5 Mar 2008

\begin{abstract}
The Fast Multipole Method allows the rapid evaluation of sums of radial basis functions centered at points distributed inside a computational domain at a large number of evaluation points to a specified accuracy $\epsilon$. The method scales as $O(N)$ in both time and memory compared to the direct method with complexity $O\left(N^{2}\right)$, which allows the solution of larger problems with given resources. Graphical processing units (GPU) are now increasingly viewed as data parallel compute coprocessors that can provide significant computational performance at low price. We describe acceleration of the FMM using the data parallel GPU architecture.

The FMM has a complex hierarchical (adaptive) structure, which is not easily implemented on dataparallel processors. We described strategies for parallelization of all components of the FMM, develop a model to explain the performance of the algorithm on the GPU architecture; and determined optimal settings for the FMM on the GPU, which are different from those on usual CPUs. Some innovations in the FMM algorithm, including the use of modified stencils, real polynomial basis functions for the Laplace kernel, and decompositions of the translation operators, are also described.

We obtained accelerations of the Laplace kernel FMM on a single NVIDIA GeForce 8800 GTX GPU in the range of 30-60 compared to a serial CPU FMM implementation. For a problem with a million sources, the summations involved are performed in approximately one second. This performance is equivalent to solving of the same problem at a 43 Teraflop rate if we use straightforward summation.
\end{abstract}

\section{Introduction}

Fast Multipole Methods (FMM) were invented in the late 1980s [8], and have since been identified as one of the ten most important algorithmic contributions in the 20th century [5]. The FMM is widely used for problems arising in diverse areas (molecular dynamics, astrophysics, acoustics, fluid mechanics, electromagnetics, scattered data interpolation etc.) because of its ability to achieve linear time and memory dense matrix vector products to a fixed prescribed accuracy $\epsilon$

Graphics Processing Units: A recent hardware trend, with their origin in the gaming and graphics industries, is the development of highly capable data-parallel processors. In 2007, while the fastest Intel CPU can only achieve 20 Gflops speeds on benchmarks, GPUs have speeds that are more than an order of magnitude higher. Of course, the GPU performs highly specialized tasks, while the CPU is a general purpose processor. Fig. 1 shows the relative abilities of GPUs and CPUs (on separate benchmarks) in early 2006. The trends reported are expected to continue for the next few years. 


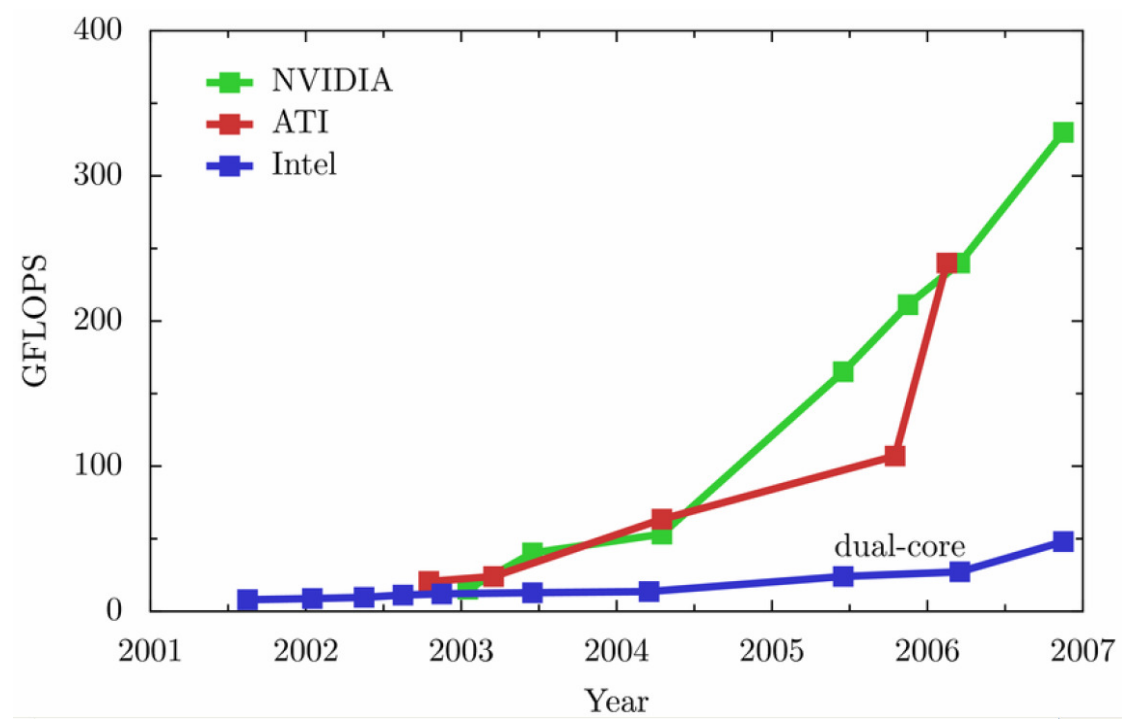

Figure 1: GPU and CPU growth in speed over the last 6 years (from [23]).

While GPUs were originally intended for specialized graphics operations, it is often possible to perform general purpose computations on them, and this has been a vigorous area of research over the last few years. One of the problems that a number of researchers have tackled for speed up is the so called $N$ body problem, in which the Coulombic (or gravitational) potentials and/or forces exerted by all charges (particles) in a system on each other are evaluated. This force calculation is part of a time stepping procedure in which the accelerations are evaluated, while the potential is necessary for energy computations. Recently several researchers have reported the use of GPUs, either in isolation or in a cluster to speed up these computations using direct algorithms, in which the interaction of every pair of particles is considered (see e.g., [18, 16, 22, 28]). While impressive speedups are reported, these algorithms suffer from their $O\left(N^{2}\right)$ nature for large $N$. Some preliminary steps towards achieving a hybrid CPU-GPU treecode are presented in work concurrent to this paper [27].

Researchers in Japan, under the auspices of the Grape project, have also sought to develop special hardware, similar to the GPU, for performing $N$ body calculations (see e.g., $[17,15]$ ). However, the substantial investments required to create special purpose hardware have meant that the progress in new generations has been slow (about 7 years since the previous Grape version was introduced). In contrast, the market driven development of the GPUs has lead to the yearly introduction of new and improved hardware.

Present contribution: We map the complex FMM algorithm on to the GPU architecture. The idea to parallelize the FMM is not new and there are publications that address some basic issues [9, 19, 20, 21, 24]. However these publications are mostly related to the development of more coarse-grained parallel algorithms. In contrast, in this paper we present algorithms that work with the nonuniform memory access costs for the GPU global and local memory, and a data parallel architecture. We use the hierarchical nature of the FMM to balance cost trees, and achieve optimal performance. This is very important as the different parts of the FMM can be accelerated with different degrees of efficiency, and at optimal settings, the overall performance is controlled not only by the slowest step of algorithm. Finally, our implementation on the GPU also suggested several improvements to the CPU FMM algorithm, which are also described.

We implemented all the parts of the FMM algorithm on the GPU and obtained a properly scaled algorithm that requires times of the order of $1 \mathrm{~s}$ to compute the matrix vector product for a million sized problem on a current NVIDIA GeForce 8800GTX GPU. This corresponds to effective flop rates of 43 Tflops, following 
the formula given in $[25,16]$.

\section{Fast multipole method (FMM)}

While several papers and books describing the FMM are available, to keep the paper self contained and to establish notation we provide a brief introduction. The basic task that the FMM performs is the computation of potentials generated by a set of sources that are evaluated at a set of evaluation points (receivers)

$$
\phi_{j}=\phi\left(\mathbf{y}_{j}\right)=\sum_{i=1}^{N} \Phi\left(\mathbf{y}_{j}, \mathbf{x}_{i}\right) q_{i}, \quad j=1, \ldots, M, \quad \mathbf{x}_{i}, \mathbf{y}_{j} \in \mathbb{R}^{d},
$$

where $\left\{\mathbf{x}_{i}\right\}$ are the sources, $\left\{\mathbf{y}_{j}\right\}$ the receivers, $q_{i}$ strengths, and $d$ is the dimensionality of the problem. The function $\Phi\left(\mathbf{y}_{j}, \mathbf{x}_{i}\right)$ is called the kernel, and the FMM has been developed for several kernels. The FMM can also be used to compute the gradient of the potential at the receiver locations as

$$
\nabla \phi_{j}=\left.\nabla_{\mathbf{y}} \phi(\mathbf{y})\right|_{\mathbf{y}=\mathbf{y}_{j}}=\left.\sum_{i=1}^{N} \nabla_{\mathbf{y}} \Phi\left(\mathbf{y}, \mathbf{x}_{i}\right)\right|_{\mathbf{y}=\mathbf{y}_{j}} q_{i} .
$$

Examples of problems where such computations arise include particle interaction, star motion, and molecular dynamics. This problem may also be recognized as a matrix vector product, where the matrix elements are derived from particular radial basis functions/potentials. Such matrices arise via the discretization of integral equation formulations of common PDE's, and iterative techniques for the solutions of the associated linear systems require repeated matrix vector products that can be accelerated via the FMM.

In this paper we will consider the Laplace kernel in 3D, for which

$$
\Phi(\mathbf{y}, \mathbf{x})=\begin{array}{ll}
|\mathbf{x}-\mathbf{y}|^{-1}, & \mathbf{x} \neq \mathbf{y} \\
0, & \mathbf{x}=\mathbf{y}
\end{array}
$$

The theory of the FMM for this kernel is well developed starting from the original works of Rokhlin and Greengard [8].

\subsection{Outline of the FMM}

The main idea of the FMM is based on performing the sum (1) via the decomposition

$$
\phi_{j}=\sum_{i=1}^{N} \Phi_{j i} q_{i}=\sum_{\mathbf{x}_{i} \notin \Omega\left(\mathbf{y}_{j}\right)} \Phi_{j i} q_{i}+\sum_{\mathbf{x}_{i} \in \Omega\left(\mathbf{y}_{j}\right)} \Phi_{j i} q_{i}, \quad j=1, \ldots, M
$$

where $\Omega\left(\mathbf{y}_{j}\right)$ is some domain (neighborhood) of the point $\mathbf{y}_{j}$. This also can be written in the matrix vector form as

$$
\phi=\boldsymbol{\Phi} \mathbf{q}=\boldsymbol{\Phi}^{(\text {dense })} \mathbf{q}+\boldsymbol{\Phi}^{(\text {sparse })} \mathbf{q} .
$$

The latter sparse matrix vector multiplication is performed directly. All other steps of the FMM are dedicated to approximate computation of $\boldsymbol{\Phi}^{(\text {dense })} \mathbf{q}$ to a specified error $\epsilon$ via the use of data structures, multipole and local expansions, and translation theory.

The algorithm can be summarized as follows. Assume that the computational domain including all sources and receivers is contained in a large cube (which can be scaled to the unit cube). This cube then is subdivided via an octree into 8 sub-cubes, and recursively going down to level $l_{\max }$, so that at each level 
we have $8^{l}$ boxes $\left(l=0, \ldots, l_{\max }\right)$. Each box containing sources can be referred as a source box and each box containing receivers can be referred as a receiver box. Obviously, depending on the distribution the $l_{\max }$ boxes can contain different numbers of points, and some boxes may even be empty. The empty boxes are skipped at all levels, which provides a basic adaptivity to the FMM for nonuniform distributions. The source boxes constitute the source hierarchy (parent-child relationships) and the receiver boxes form the receiver box hierarchy. The structuring of the initial source and receiver boxes can be done using spatial ordering (bit-interleaving technique, e.g. see [11]). This technique also allows one to determine neighbors for each box, which is important for the FMM. Also some necessary computations can be done before the run of the FMM (for example, precomputation of the elements of the translation matrices).

The FMM itself computes $\phi_{j}$ and $\nabla \phi_{j}$ for an arbitrary input vector $\left\{q_{i}\right\}$. This part can be repeated for the same source and receiver locations many times (e.g. for iterative solution of linear system (1), when the solution $\left\{q_{i}\right\}$ must be found for a given $\left\{\phi_{j}\right\}$ ). The FMM consists of three basic steps: Upward pass, Downward Pass, and Final Summation, which are described in detail below.

\subsubsection{Upward Pass}

Step 1 For each box at the finest level $l_{\max }$, generate a multipole, or $S$ expansion for each source at the box center. This expansion is vector of expansion coefficients $\left\{C_{n}^{m}\right\}$ over some basis (usually the multipole solutions of Laplace's equation). As function representation in this basis is infinite, the series are truncated with truncation number $p$, so that there are $p^{2}$ coefficients associated with each box center. The choice of $p$ is dictated by the required accuracy. The maximum achievable accuracy on any computer architecture is related to the machine precision (single or double precision), and this provides a limit on the maximum $p$ that may be used. The expansions from all sources in the box are consolidated in a single expansion by summing the coefficients corresponding to each source.

Step 2 Moving up the source hierarchy, for levels from $l_{\max }$ to 2 , we generate $S$ expansion coefficients for each box from the coefficients of its children. This is done using the multipole-to-multipole (or $S \mid S$ ) translation operator on the coefficients of each child box, and consolidating (summing) the results. The procedure goes in a hierarchical way from level to level.

\subsubsection{Downward Pass}

Starting from level 2 down to level $l_{\max }$ for each receiver box generate its local, or $R$ expansion. This is achieved via the following two step procedure:

Step 1 Translate the multipole $S$ expansions from the source boxes at the same level belonging to the neighborhood of the receiver box's parent, but not belonging to the neighborhood of the receiver box itself, to $R$ expansions centered at the center of the receiver box using multipole-to-local, or $S \mid R$, translations. Consolidate all the expansions into one coefficient vector.

Step 2 Translate the $R$ expansion coefficients from the parent receiver box center to child box centers and consolidate them with the $S \mid R$ translated expansions at this level available from Step 1. This step starts from level 3 (or formally from level 2, if the $R$ expansion for the parent box is considered initialized to zero).

\subsubsection{Final Summation}

Step 1 Evaluate local $R$ expansions for each receiver box at level $l_{\max }$ at all receiver locations in the box. 
Step 1' If gradient computations are needed, apply the sparse matrices corresponding to differentiation to the vectors of expansion coefficients, and efficiently obtain the expansion coefficients for the gradient. Evaluate the gradient expansion at each receiver location in the box.

Step 2 For each receiver, perform a direct summation of the sources belonging to its box neighborhood and sum this with the result of Step 1 and Step 1' of the Final summation. This step is nothing but a sparse matrix vector multiplication (or direct evaluation). Note that the sparse matrix vector product is independent of the rest of the FMM and can be performed separately.

This also can be represented as a flow chart 2 .

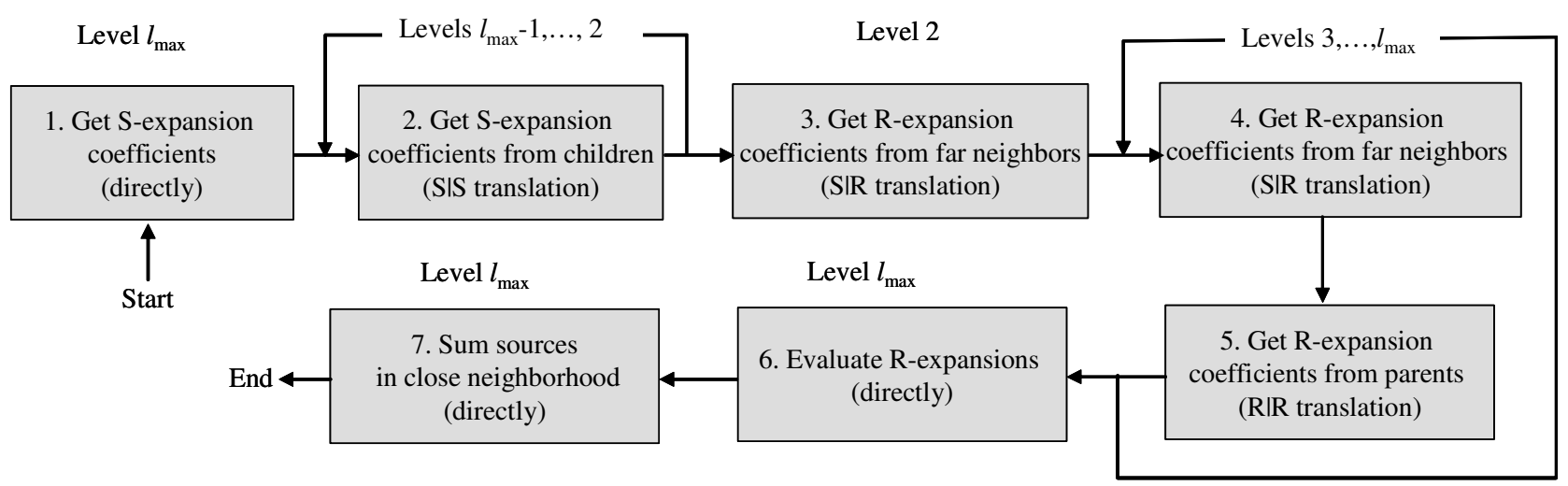

Figure 2: A flow chart of the standard FMM.

\subsection{Characteristics of our FMM implementation}

There are several bases, translation, methods and schemes for the FMM for the Laplace kernel. Each of these have their own advantages and disadvantages. The choice of a particular method depends on the problem to be solved and the accuracy desired. We consider here an FMM algorithm consistent with the single precision floating point arithmetic on the GPU. While some software for double precision exists on the GPU, their use is reported to show a decrease of the computational speed by up to 10 times (see the discussion e.g., in [7]), and while we will still see an acceleration relative to the CPU, this will not be as dramatic. GPU manufacturers envision in the closest future the release of GPUs with double precision hardware (both ATI and NVIDIA have announced that this feature will be released in 2008). In this case, single precision algorithms can be modified accordingly and the fastest methods for high precision computations can be implemented and tested, without writing artificial libraries.

Thus, for the current state of the GPU computations with 3,4, or 5 digit accuracy are appropriate, which cover a broad class of practical needs. Our tests showed that computations with a truncation number $p=16$ and higher using 4 byte floats produce a heavy loss of accuracy, overflows/underflows (due to summation of numbers of very different magnitude) and cannot be used for large scale problems. On the other hand, computations with relatively small truncation numbers, like $p=4,8$, and 12 are stable, and produced the required 3, 4 or 5 digits for problems with $N \simeq 10^{6}$, and we focus on this range of truncation numbers in this paper.

Our comparative study of various translation methods on the CPU [13] show that for this range, the standard multipole expansions and rotation-coaxial translation-rotation (RCR) decomposition of the translation operators provide the best results. The RCR decomposition introduced for the FMM in Ref. [26] has 
$O\left(p^{3}\right)$ complexity with low asymptotic constant for any translation operator. For the values of $p$ considered here, they are as fast or faster than the $O\left(p^{2}\right)$ methods in the literature, which have much larger asymptotic constants and result in larger sizes of the representing vectors [10].

We also revised and modified the computationally expensive original $S \mid R$ translation scheme associated with the conventional FMM, and used normalized real basis functions that can be evaluated quickly on the GPU.

\subsubsection{Basis functions}

In spherical coordinates $(r, \theta, \varphi)$

$$
x=r \sin \theta \cos \varphi, \quad x=r \sin \theta \sin \varphi, \quad z=r \cos \theta
$$

elementary solutions of the Laplace equation in 3D can be represented as

$$
R_{n}^{m}(\mathbf{r})=\alpha_{n}^{m} r^{n} Y_{n}^{m}(\theta, \varphi), \quad S_{n}^{m}(\mathbf{r})=\beta_{n}^{m} r^{-n-1} Y_{n}^{m}(\theta, \varphi), \quad n=0,1, \ldots, \quad m=-n, \ldots, n .
$$

Here $R_{n}^{m}(\mathbf{r})$ are the regular (local) spherical basis functions and $S_{n}^{m}(\mathbf{r})$ the singular (far field, or multipole) spherical basis functions; $\alpha_{n}^{m}$ and $\beta_{n}^{m}$ are normalization constants which can be selected by convenience, and $Y_{n}^{m}(\theta, \varphi)$ are the orthonormal spherical harmonics:

$$
\begin{aligned}
Y_{n}^{m}(\theta, \varphi) & =N_{n}^{m} P_{n}^{|m|}(\mu) e^{i m \varphi}, \quad \mu=\cos \theta \\
N_{n}^{m} & =(-1)^{m} \sqrt{\frac{2 n+1}{4 \pi} \frac{(n-|m|) !}{(n+|m|) !}}, \quad n=0,1,2, \ldots, \quad m=-n, \ldots, n,
\end{aligned}
$$

where $P_{n}^{|m|}(\mu)$ are the associated Legendre functions [2]. We will use the definition of the associated Legendre function $P_{n}^{m}(\mu)$ that is consistent with the value on the cut $(-1,1)$ of the hypergeometric function $P_{n}^{m}(z)$ (see Abramowitz \& Stegun, [2]). These functions can be obtained from the Legendre polynomials $P_{n}(\mu)$ via the Rodrigues' formula

$$
P_{n}^{m}(\mu)=(-1)^{m}\left(1-\mu^{2}\right)^{m / 2} \frac{d^{m}}{d \mu^{m}} P_{n}(\mu), \quad P_{n}(\mu)=\frac{1}{2^{n} n !} \frac{d^{n}}{d \mu^{n}}\left(\mu^{2}-1\right)^{n} .
$$

Straightforward computation of these basis functions involves several relatively costly operations, such as conversion back and forth to spherical coordinates. Further, as defined above, these functions are complex. This is an unnecessary expense when the terms in (1) are all real.

One of the most convenient bases for translation was proposed by Epton and Dembart [6], which is basis (7) with

$$
\begin{aligned}
& \alpha_{n}^{m}=(-1)^{n} i^{-|m|} \sqrt{\frac{4 \pi}{(2 n+1)(n-m) !(n+m) !}}, \\
& \beta_{n}^{m}=i^{|m|} \sqrt{\frac{4 \pi(n-m) !(n+m) !}{2 n+1}}, \quad n=0,1, \ldots, \quad m=-n, \ldots, n .
\end{aligned}
$$

However, these functions are also complex. To obtain a basis of real functions we instead set

$$
\begin{aligned}
\alpha_{n}^{m} & =(-1)^{n} \sqrt{\frac{4 \pi}{(2 n+1)(n-m) !(n+m) !}}, \quad \beta_{n}^{m}=\sqrt{\frac{4 \pi(n-m) !(n+m) !}{2 n+1}}, \\
n & =0,1, \ldots ., \quad m=-n, \ldots, n .
\end{aligned}
$$


and define real basis functions as

$$
\widetilde{R}_{n}^{m}=\left\{\begin{array}{ll}
\operatorname{Re}\left\{R_{n}^{m}\right\}, & m \geqslant 0 \\
\operatorname{Im}\left\{R_{n}^{m}\right\}, & m<0
\end{array}, \quad \widetilde{S}_{n}^{m}=\left\{\begin{array}{ll}
\operatorname{Re}\left\{S_{n}^{m}\right\}, & m \geqslant 0 \\
\operatorname{Im}\left\{S_{n}^{m}\right\}, & m<0
\end{array} .\right.\right.
$$

In these bases the regular (local) and singular (multipole) expansions of harmonic functions take the form

$$
\phi(\mathbf{r})=\sum_{n=0}^{p-1} \sum_{m=-n}^{n} D_{n}^{m} \widetilde{R}_{n}^{m}(\mathbf{r}), \text { or } \phi(\mathbf{r})=\sum_{n=0}^{p-1} \sum_{m=-n}^{n} C_{n}^{m} \widetilde{S}_{n}^{m}(\mathbf{r}),
$$

where the $C_{n}^{m}$ and $D_{n}^{m}$ coefficients are real, and the infinite sums are truncated and $p^{2}$ terms retained.

Note that in an actual FMM implementation evaluation of the singular basis functions can be avoided, since the evaluation stage requires only the regular basis function, while the coefficients of the multipole expansion over the singular basis are the normalized regular basis functions,

$$
G\left(\mathbf{r}, \mathbf{r}_{0}\right)=\frac{1}{\left|\mathbf{r}-\mathbf{r}_{0}\right|}=\sum_{n=0}^{\infty} \sum_{m=-n}^{n}(-1)^{n} R_{n}^{-m}\left(\mathbf{r}_{0}\right) S_{n}^{m}(\mathbf{r}) .
$$

Dropping details of derivation, one can compute $\widetilde{R}_{n}^{m}(\mathbf{r})$ using the following recursions

$$
\begin{aligned}
& \widetilde{R}_{0}^{0}=1, \quad \widetilde{R}_{1}^{1}=-\frac{1}{2} x, \quad \widetilde{R}_{1}^{-1}=\frac{1}{2} y, \\
& \widetilde{R}_{|m|}^{|m|}=-\frac{\left(x \widetilde{R}_{|m|-1}^{|m|-1}+y \widetilde{R}_{|m|-1}^{-|m|+1}\right)}{2|m|}, \quad \widetilde{R}_{|m|}^{-|m|}=\frac{\left(y \widetilde{R}_{|m|-1}^{|m|-1}-x \widetilde{R}_{|m|-1}^{-|m|+1}\right)}{2|m|}, \quad|m|=2,3, \ldots . \\
& \widetilde{R}_{|m|+1}^{m}=-z \widetilde{R}_{|m|}^{m}, \quad m=0, \pm 1, \ldots . \\
& \widetilde{R}_{n}^{m}=-\frac{(2 n-1) z \widetilde{R}_{n-1}^{m}+r^{2} \widetilde{R}_{n-2}^{m}}{(n-|m|)(n+|m|)}, \quad n=|m|+2, \ldots, \quad m=-n, \ldots, n .
\end{aligned}
$$

These recursions show that functions $\widetilde{R}_{n}^{m}$ are real polynomials of degree $n$ of Cartesian coordinates of $\mathbf{r}=(x, y, z)$. To compute them there is no need to convert to spherical coordinates and back. Further, their use avoids the need to use special functions, which might be implemented with limited accuracy and longer clock cycles, a consideration that is important on both the GPU and the CPU. Finally, the algorithm does not need complex arithmetic.

\subsubsection{RCR decomposition}

Elementary regular and multipole (singular) solutions of the Laplace equation centered at a point can be expanded in series over elementary solutions centered about some other spatial point. This can be written as the following addition theorems

$$
\begin{aligned}
& R_{n}^{m}(\mathbf{r}+\mathbf{t})=\sum_{n^{\prime}=0}^{\infty} \sum_{m^{\prime}=-n^{\prime}}^{n^{\prime}}(R \mid R)_{n^{\prime} n}^{m^{\prime} m}(\mathbf{t}) R_{n^{\prime}}^{m^{\prime}}(\mathbf{r}), \\
& S_{n}^{m}(\mathbf{r}+\mathbf{t})=\sum_{n^{\prime}=0}^{\infty} \sum_{m^{\prime}=-n^{\prime}}^{n^{\prime}}(S \mid R)_{n^{\prime} n}^{m^{\prime} m}(\mathbf{t}) R_{n^{\prime}}^{m^{\prime}}(\mathbf{r}), \quad|\mathbf{r}|<|\mathbf{t}| \\
& S_{n}^{m}(\mathbf{r}+\mathbf{t})=\sum_{n^{\prime}=0}^{\infty} \sum_{m^{\prime}=-n^{\prime}}^{n^{\prime}}(S \mid S)_{n^{\prime} n}^{m^{\prime} m}(\mathbf{t}) S_{n^{\prime}}^{m^{\prime}}(\mathbf{r}), \quad|\mathbf{r}|>|\mathbf{t}|
\end{aligned}
$$


where $\mathbf{t}$ is the translation vector, and $(R \mid R)_{n^{\prime} n}^{m^{\prime} m},(S \mid R)_{n^{\prime} n}^{m^{\prime} m}$, and $(S \mid S)_{n^{\prime} n}^{m^{\prime} m}$ are the four index local-tolocal, multipole-to-local, and multipole-to-multipole reexpansion coefficients. Explicit expressions for these coefficients can be found elsewhere (see e.g. $[6,4]$ ). It is not difficult to show then that reexpansion of the basis produces the entries of the matrix translation operator, which relates expansion coefficients in different bases with different centers. For example for multipole-to-local translation we have

$$
D_{n}^{m}=\sum_{n^{\prime}=0}^{\infty} \sum_{m^{\prime}=-n^{\prime}}^{n^{\prime}}(S \mid R)_{n n^{\prime}}^{m m^{\prime}}(\mathbf{t}) C_{n^{\prime}}^{m^{\prime}}
$$

where $C_{n^{\prime}}^{m^{\prime}}$ are coefficients of multipole expansion in some reference frame, and $D_{n}^{m}$ are coefficients of expansion of the same function over the local basis in the reference frame with the origin shifted by the translation vector $\mathbf{t}$.

Despite the fact that the coefficients of the translation matrix can be easily computed, the translation operation converting $p^{2}$ to $p^{2}$ coefficients requires $p^{4}$ multiplications if applied directly, which is expensive. This can be reduced to $2 p^{3}+O\left(p^{2}\right)$ for the $S \mid R$ translation using the RCR decomposition (see [26] for an initial treatment, and $[11,13]$ for extensions). In this decomposition we first perform rotation of the reference frame to point the $z$ axis towards the target, then perform coaxial translation along the rotated $z$ axis, and then rotate the reference frame back (see Fig. 3). Each operation has $O\left(p^{3}\right)$ complexity due to acts only on certain subspaces of the coefficient vector.

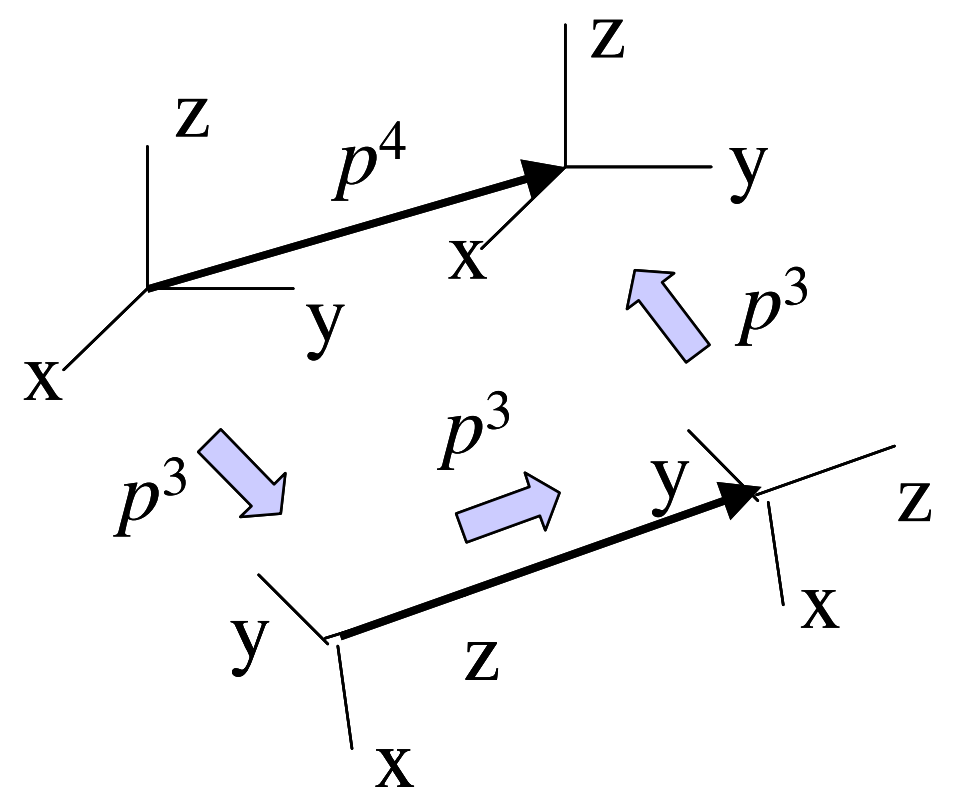

Figure 3: Illustration of the rotation coaxial-translation rotation (RCR) technique for $O\left(p^{3}\right)$ translation (see [13]).

For coaxial translations we have

$$
(S \mid R)_{n n^{\prime}}^{m m^{\prime}}\left(\mathbf{i}_{z} t\right)=\delta_{m m^{\prime}}(S \mid R)_{n n^{\prime}}^{m}(t)
$$

where $\delta_{m m^{\prime}}$ is the Kronecker symbol and expressions for the $S \mid S$ and $R \mid R$ translations are similar. In the 
basis we use, the entries of the coaxial translation matrices are

$$
\begin{aligned}
& (R \mid R)_{n n^{\prime}}^{m}(t)=r_{n^{\prime}-n}(t), \quad n^{\prime} \geqslant|m|, \\
& (S \mid R)_{n n^{\prime}}^{m}(t)=s_{n+n^{\prime}}(t), \quad n, n^{\prime} \geqslant|m|, \\
& (S \mid S)_{n n^{\prime}}^{m}(t)=r_{n-n^{\prime}}(t), \quad n \geqslant|m|,
\end{aligned}
$$

where the functions $r_{n}(t)$ and $s_{n}(t)$ are

$$
r_{n}(t)=\frac{(-t)^{n}}{n !}, \quad s_{n}(t)=\frac{n !}{t^{n+1}} \quad n=0,1, \ldots, \quad t \geqslant 0
$$

and zero for $n<0$.

The rotation transform is a bit more tricky, since in the normalized basis the rotation operators are different in the $S$ and $R$ bases. However multiplication of the rotation operators by diagonal matrices makes them the same in the bases, and this can be used for more compact storage of the rotation data. The diagonals of these diagonal matrices consist of coefficients $\alpha_{n}^{m}$ for the $R$ basis and $\beta_{n}^{m}$ for the $S$ basis (which is equivalent to the use of bases (7) with $\alpha_{n}^{m}=1$ and $\beta_{n}^{m}=1$ ). Note that in this case the rotation operators are related to rotation of spherical harmonics. A detailed theory of fast recursive computation of the rotation coefficients and rotation can be found e.g. in our book [11] or in our technical report [13]. We use the methods described there with small modifications to account for the fact that we deal with the purely real basis functions described above.

\subsubsection{Translation stencil}

One of the reasons for the high cost of the FMM multipole-to-local translation stage in the original algorithm is that there are 189 translations per non boundary receiver box in 3D. In [4] more efficient translation operators for large $p$ based on an exponential representation and a translation stencil that took advantage of symmetries and reduced the number of translations to 40 were introduced. As discussed earlier, for the accuracies that can be achieved on the GPU, the usual FMM representation in terms of multipoles and regular functions, along with the $O\left(p^{3}\right)$ translation operators are preferred. We found some symmetries that allow the translations in the regular FMM stencil to be reduced.

Consider the neighborhood of the parent receiver box. There are 216 boxes on the level corresponding to that of the receiver box. $S$ expansions from boxes that are not in the neighborhood of the receiver box should be translated to $R$ expansions at each box center. Considering the 8 children boxes together, we have $8 \times 189=1512$ translations. We observe that there exist 80 boxes (see the 3D parent neighborhood in Fig. 4) from which the $S \mid R$ translations can be made not to the children, but to the parent box itself, while still satisfying the specified error bound. Such translations can be consolidated with the $R$ expansion at the parent box and Step 2 of the downward pass can be performed without any additional constraint. The saving here is that instead of $80 \times 8=640$ translations (each source box to each receiver box) we have only 80 translations, which saves 560 translations out of 1512 for this domain, or effectively reduces 189 translations per box to 119 translations.

This is an example of a simple translation stencil, which amortizes the translation cost for boxes having the same parent. On the CPU we implemented and tested an even more advanced translation stencil which reduces this number of translations by another factor of 2 (more precisely, to $61 S \mid R$ translations per receiver box). However that stencil requires a more complicated internal data structure (domains composed of 2 and 4 children cubes) and the GPU code runs a bit slower than the one for the stencil described above, due to more extensive global memory access. In the implementation described in this paper we limited ourselves with the above simplification. 


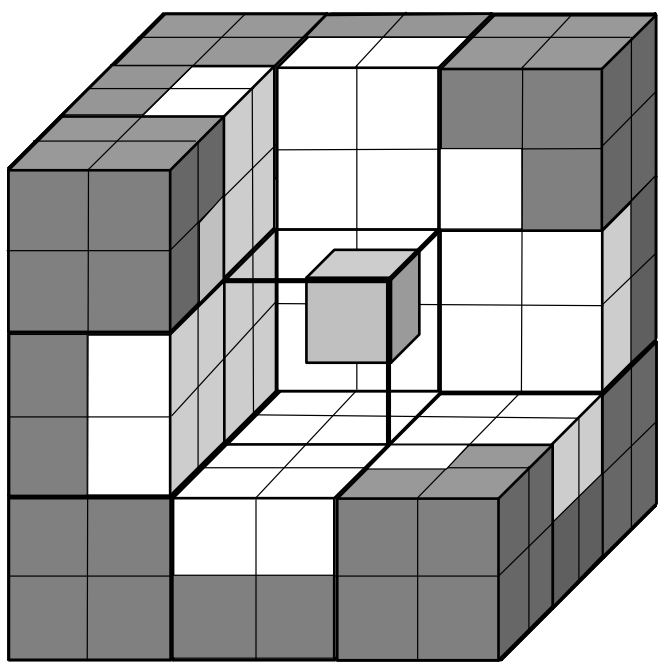

Figure 4: A section of the translation stencil used in the computations. Instead of translation to the central receiver box (the light gray shade) the source boxes shown in the dark gray can be translated to the center of the parent receiver box (the center of the stencil). Then this is information can be passed to each child box.

A theoretical justification of this scheme follows from the error bounds provided below. Introduction of the stencil modifies the 2-step downward pass of the FMM as follows (the white source boxes in Fig. 4 are referred as boxes of type 1, while the shaded source boxes are boxes of type 2).

Modified Downward Pass:

Starting from level 2 down to level $l_{\max }$ for each receiver box generate its local, or $R$ expansion. This is achieved by the following procedure:

Step 1. Translate $S$ expansions from the source boxes of type 1 belonging to the receiver stencil to the $R$ expansions centered at the center of the receiver box (multipole-to-local, or $S \mid R$ translations). Consolidate the expansions.

Step 2. Translate $S$ expansions from the source boxes of type 2 belonging to the receiver stencil to the $R$ expansions centered at the center of the parent receiver box. Consolidate the expansions with the existing parent $R$ expansion (zero for level 2).

Step 3. Translate the $R$ expansion from the parent receiver box and consolidate with the $S \mid R$ translated expansions.

\subsubsection{Variable truncation number}

In the standard FMM for the Laplace equation the truncation number $p$ is fixed for all translations/expansions. The choice of $p$ is dictated by the error bound evaluated for the "worst" case, which corresponds to the $S \mid R$ translation from the source box of size 1 to the receiver box of size 1 whose centers are separated by a distance of 2 box units (the Laplace equation is scale independent). Let us provide a bit more general look at the problem.

Assume that a set of sources is located within a $d$ dimensional sphere of radius $R_{1}$, which we denote as $\Omega_{1}$ and the set of evaluation points is located within a $d$ dimensional sphere of radius $R_{2}$, which we denote as $\Omega_{2}$. We assume that these domains do not intersect and denote the distance between the centers of these spheres as $D>R_{1}+R_{2}$. Assume that the multipole expansions about the center of $\Omega_{1}$ converges absolutely 


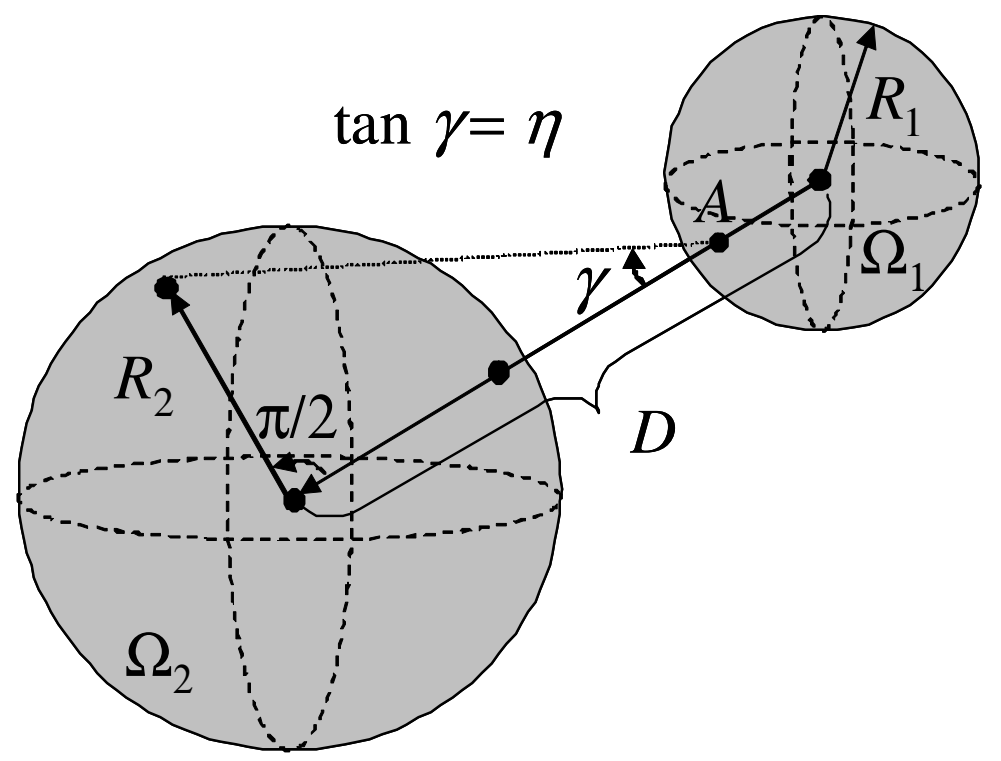

Figure 5: Illustration of the $\mathrm{S} \mid \mathrm{R}$ translation from source domain $\Omega_{1}$ of radius $R_{1}$ to receiver domain $\Omega_{2}$ of radius $R_{2}$ which centers are separated by distance $D$. The parameter $\eta$ can be interpreted as the tangent of angle $\gamma$ shown in the figure, where $A$ is the point of $\Omega_{1}$ closest to $\Omega_{2}$.

and uniformly and the same holds for the local expansions about the center of $\Omega_{2}$. For the Laplace equation these series can be majorated by geometric progressions. More precisely, if the series are $p$ truncated, then the error, $\epsilon_{p}$, is bounded as

$$
\epsilon_{p}<C \eta^{p}, \quad \eta\left(\Omega_{1}, \Omega_{2}\right)=\frac{\max \left(R_{1}, R_{2}\right)}{D-\min \left(R_{1}, R_{2}\right)},
$$

where $C$ is some constant. Note that $\eta\left(\Omega_{1}, \Omega_{2}\right)$ is a purely geometrical quantity (see Fig. 5), which characterizes the multipole-to-local translation. If $\eta \geqslant 1$ this means that the intersection of domains $\Omega_{1}$ and $\Omega_{2}$ is not empty and the expansions diverge. The error due to translation of the expansion of a single source will be a function of $\eta$ and for a given $\eta<1$ we can determine the number of terms to guarantee the required accuracy. A larger $\eta$, means that more terms are needed for a specified error.

The FMM errors for the kernels under consideration are determined by the errors of the multipole-tolocal translations. For the kernels considered, when the same length series (truncation numbers) can be used for all types of translations for all levels, the only criteria, which needs to be checked to stay within the specified error bounds is

$$
\eta\left(\Omega_{1}, \Omega_{2}\right) \leqslant \eta_{*}(d) .
$$

The quantity $\eta_{*}$ depends on the dimension $d$. In the standard translation scheme, the distance between the centers of the closest domains (or centers of the cubic boxes of unit size separated by one box) is $D=2$, while the radii of the minimal spheres which include the source and receiver boxes are $R_{1}=R_{2}=d^{1 / 2} / 2$. So the standard $\eta_{*}$ which determines the length of expansion for a specified error is

$$
\eta_{*}(d)=\frac{d^{1 / 2}}{4-d^{1 / 2}}, \quad \eta_{*}(3) \approx 0.7637 .
$$

The translation stencil scheme suggested above then can be justified, as in this case we have $R_{2}=3^{1 / 2}$, $R_{1}=3^{1 / 2} / 2$ and one can check directly that any gray box in Fig. 4 has a distance from the stencil center 
greater than

$$
D_{*}=\min \left(R_{1}, R_{2}\right)+\frac{\max \left(R_{1}, R_{2}\right)}{\eta_{*}(3)} .
$$

Consider now the general case that includes both the white and gray boxes in Fig. 4. To provide the same error as for the closest boxes, it is sufficient to use the truncation number

$$
p^{\prime}=\frac{\ln \epsilon_{p}-\ln C}{\ln \eta}
$$

which follows from the first Eq. (20). On the other hand for given $p$ we have

$$
p=\frac{\ln \epsilon_{p}-\ln C}{\ln \eta_{*}} .
$$

Therefore, the truncation number providing the same error can be determined from

$$
p^{\prime}=p \frac{\ln \eta_{*}}{\ln \eta}=p \ln \eta_{*} / \ln \frac{\max \left(R_{1}, R_{2}\right)}{D-\min \left(R_{1}, R_{2}\right)} .
$$

As parameters $p, \eta_{*}$, and $R_{1}$ are fixed we can for any $S \mid R$ translation determine the effective truncation number $p^{\prime}$, which does not exceed $p$ and, therefore the function representation of length $p^{\prime 2}$ can be obtained from the similar representation of length $p^{2}$ by a simple additional truncation. We found that such additional truncations bring substantial computational savings without substantial loss of accuracy.

Remark 1 In fact this algorithm is to be preferred in the sense that the errors actually achieved are closer to the specified error $\epsilon$ (while not exceeding it); and wasteful extra precision computations are not performed.

Remark 2 Translation with reduced $p$ means use of rectangularly truncated translation matrices $p^{2} \times p^{\prime 2}$ which act on vectors of size $p^{2}$ and produce vectors of size $p^{2}$.

\subsection{Computation of gradient}

As soon as expansion coefficients $D_{n}^{m}$ for a given box are found, we have for the gradient of the far field (see the first Eq. (13)):

$$
\nabla \phi(\mathbf{r})=\sum_{n=0}^{p-1} \sum_{m=-n}^{n} D_{n}^{m} \nabla \widetilde{R}_{n}^{m}(\mathbf{r})+\operatorname{error}(p)=\sum_{n=0}^{p-1} \sum_{m=-n}^{n} \mathbf{D}_{n}^{m} \widetilde{R}_{n}^{m}(\mathbf{r})+\operatorname{error}(p)
$$

where $\mathbf{D}_{n}^{m}$ is a real three-dimensional vector, whose $x, y$, and $z$ components correspond to the components of the gradient. It is clear that such a representation should hold since derivatives of a harmonic functions are also harmonic functions expandable over the same basis. Explicit expressions for $\mathbf{D}_{n}^{m}$ can be obtained from the recursions for local basis functions $R_{n}^{m}$ - a method described in [11]. Such recursions can be found elsewhere (e.g. $[6,12])$. Note however, that one should convert these formulae to the real basis according to the definitions (7), (11), and (12). 


\subsection{Computational complexity}

The relative costs of the different steps of the FMM depend on the source and receiver distributions, truncation number $p$ (or accuracy of computations), and on $l_{\max }$. To simplify the analysis and provide benchmark results we constrain ourselves to cases where the $N$ sources and $M$ receivers are randomly (uniformly) distributed inside a unit cube (usually we selected $M$ to be of the same order of magnitude as $N$, so that in the complexity estimates one can assume $M \approx N$ ).

The main optimization of the FMM is based on proper selection of the depth of the octree, or $l_{\max }$. For a given size of the problem, $N$, it is convenient to introduce the clustering parameter, $s$, which is the number of sources (and for our case receivers) in the smallest box. In this case the computational costs for the sparse and dense matrices are

$$
\operatorname{Cost}^{(\text {dense })}=N\left(A_{1}+\frac{A_{2}}{s}\right), \quad \operatorname{Cost}^{(\text {sparse })}=B_{1} N s, \quad s=N \cdot 8^{-l_{\max }},
$$

where $A_{1}$ and $A_{2}$ are some constants depending on the translation scheme and prescribed accuracy, $B_{1}$ is a constant determined by the size of the neighborhood and the computational complexity of function $\Phi(\mathbf{y}, \mathbf{x})$. The cluster size $s$ must be chosen to minimize the overall algorithm cost.

If the algorithm is implemented on a single processor the CPU time required for execution of the task can be evaluated as

$$
\operatorname{Time}^{(\text {sngl })}=C \cdot\left(\operatorname{Cost}^{(\text {dense })}+\operatorname{Cost}^{(\text {sparse })}\right)=C N\left(A_{1}+\frac{A_{2}}{s}+B_{1} s\right),
$$

where $C$ is some constant, and the optimal clustering parameter and maximum level of space subdivision can be found easily by differentiation of this function with respect to $s$ :

$$
s_{\text {opt }}=\left(\frac{A_{2}}{B_{1}}\right)^{1 / 2}, \quad l_{\max }^{(o p t)}=\log _{8} \frac{N}{s_{\text {opt }}} .
$$

This provides an optimal time

$$
\text { Time }_{\text {opt }}^{(\text {sngl })}=C N\left[A_{1}+2\left(A_{2} B_{1}\right)^{1 / 2}\right],
$$

which also shows that in the case when constants $A, B$, and $C$ do not depend on $N$, the optimal algorithm scales as $O(N)$.

Note that normally $A_{1} \ll\left(A_{2} B_{1}\right)^{1 / 2}$ ( $A_{1}$ is determined by the Step 1) of the Upward Pass and Step 1) of Final Summation, while most computational work in the FMM is related to the $S \mid R$ translations). In this case the optimal settings and the total optimal time depend on $A_{2}, B_{1}$ and the processor $(C)$.

\section{NVIDIA G80 series GPU Architecture}

The NVIDIA G80 GPU, the one on which we developed our software, is the current generation of NVIDIA GPU, and has also been released as the Tesla compute coprocessor. It consists of a set of multiprocessors (16 on our GeForce 8800 GTX), each composed of 8 processors. All multiprocessors talk to a global device memory, which in the case of our GPU is $768 \mathrm{MB}$, but can be as large as $1.5 \mathrm{~GB}$ for more recently released GPUs/coprocessors. The 8 processors in each multiprocessor share $16 \mathrm{kB}$ local read-write "shared" memory, a local set of 8192 registers, and a constant memory of $64 \mathrm{kB}$ over all multiprocessors, of which $8 \mathrm{kB}$ can be cached locally at one multiprocessor.

A programming model (Compute Unified Device Architecture or CUDA) and a C compiler (with language extensions) that compiles code to run on the GPU are provided by NVIDIA. This model is supposed 
to be extended over the next few generations of processors, making investment of effort on programming it worthwhile. Under CUDA the GPU is a compute device that is a highly multithreaded coprocessor. A thread block is a batch of threads that executes on a multiprocessor that have access to its local memory. They perform their computations and become idle when they reach a synchronization point, waiting for other threads in the block to reach the synchronization point. Each thread is identified by its thread ID (one, two or three indices). The choice of 1,2 or 3D index layout is used to map the different pieces of data to the thread. The programmer writes data parallel code, which executes the same instructions on different data, though some customization of each thread is possible based on different behaviors depending on the value of the thread indices.

To achieve efficiency on the GPU, algorithm designers must account for the substantially higher cost (two orders of magnitude higher) to access fresh data from the GPU main memory. This penalty is paid for the first data access, though additional contiguous data in the main memory can be accessed cheaply after this first penalty is paid. An application that achieves such efficient reads and writes to contiguous memory is said to be coalesced. Thus programming on the nonuniform memory architecture of the GPU requires that each of the operations be defined in a way that ensures that main memory access (reads and writes) are minimized, and coalesced as far as possible when they occur.

We have developed an extensible middleware library [14] that works with NVIDIA's CUDA to ease the burden of programming on the GPU, and allows writing code in Fortran 9x. The FMM was programmed using this library and a few functions written in NVIDIA's extended C.

\section{FMM on GPU}

Peculiarities of the architecture, memory constraints and bandwidth, and relative costs of the memory access and various arithmetic operations drastically change the complexity model and cost balance of the FMM on GPU compared with that on CPU. Our study shows that on the GPU the performance is limited mostly by some intrinsic balance for efficient sparse matrix vector multiplication, and in lesser degree by the balance of the $S \mid R$ translations and direct summations, which in contrast is the sole criterion for optimal performance on the CPU.

\subsection{Direct summation baseline}

First, let us consider a direct method for solution of problem (1), i.e. computation of the sum without the FMM as a baseline for comparison. In examples of efficient use of CUDA the NVIDIA provides a routine for dot product, which can be used as a part of a routine implementing the matrix vector product. However, our experiments with this routine showed that it is actually designed for a given, or precomputed matrix. (The same remark applies to the CUDA provided SGEMV routine). This is not the case in this problem where the matrix entries are computed "on the fly" as needed, which does not require large storage or additional memory access.

Our algorithm is designed in the following way.

1. All source data, including coordinates and source intensities are stored in a single array in the global GPU memory (the size of the array is $4 N$ for potential evaluation and $7 N$ for potential/force evaluation). Two more large arrays reside in the global memory, one with the receiver coordinates (size $3 M$ ), and the other is allocated for the result of the matrix vector product ( $M$ for potential computations and $4 M$ for potential and force computations). In case the sources and receivers are the same, as in particle dynamics computations, these can be reduced.

2. The routine is executed in CUDA on a one dimensional grid of one dimensional blocks of threads. By default each block contains 256 threads, which was obtained via an empirical study of optimal thread- 
block size. This study also showed that for good performance the thread block grid should contain not less than 32 blocks for a 16 multiprocessor configuration. If the number of receivers is not a divisor of the block size, only the remaining number of threads is employed for computations of the last block.

3. Each thread in the block handles computation of one element of the output vector (or one receiver). For this purpose a register for potential calculation (and three registers for calculation of forces) are allocated and initialized to zero. The thread can take care on another receiver only after the entire block of threads is executed, threads are synchronized, and the shared memory can be overwritten.

4. Each thread reads the respective receiver coordinates directly from the global memory and puts them into the thread stack.

5. For a given block the source data are executed in a loop by batches of size $B$ floats, where $B$ depends on the type of the kernel (4 or 7 floats per source for monopole or monopole/dipole summations, respectively) and the size of the shared memory available. Currently we use $8 \mathrm{kB}$, or 2048 floats of the shared memory (so e.g. for monopole summation this determines $B=512$ ). If the number of sources is not a divisor of $B$ the last batch takes care of the remaining number of sources.

6. All threads of the block are employed to read source data for the given batch from global memory and put them into the shared memory (consequent reading: one thread per one float, until the entire batch is loaded followed by thread synchronization).

7. Each thread computes product of one matrix element (kernel) with the source intensity (or in the case of dipoles the dipole moment with the kernel) and sums it up with the content of the respective summation register.

8. After summation of contributions of all sources (all batches) each thread writes the sum from its register into the global memory.

\begin{tabular}{|l|l|l|l|l|l|l|}
\hline \multicolumn{7}{|c|}{ Table 1: Comparison of direct computations on the CPU \& GPU } \\
Exponents in brackets; italics indicate estimates rather than computations \\
\hline & \multicolumn{3}{|c|}{ Potential } & \multicolumn{3}{c|}{ Potential+ Force } \\
\hline$N$ & CPU (s) & GPU(s) & ratio & CPU(s) & GPU(s) & ratio \\
209 \\
1024 & $5.015 \mathrm{E}-2$ & $3.246 \mathrm{E}-4$ & 154 & 0.102 & $4.86(-4)$ & 209 \\
\hline 2048 & $2.017 \mathrm{E}-1$ & $7.959 \mathrm{E}-4$ & 253 & 0.4095 & $9.53(-4)$ & 429.5 \\
\hline 4096 & $7.986 \mathrm{E}-1$ & $2.201 \mathrm{E}-3$ & 362 & 1.633 & $2.99(-3)$ & 546 \\
\hline 8192 & 3.185 & $6.851 \mathrm{E}-3$ & 465 & 6.541 & $9.86(-3)$ & 663 \\
\hline 16384 & 12.764 & $2.424 \mathrm{E}-2$ & 527 & 26.155 & $3.49(-2)$ & 750 \\
\hline 32768 & 51.06 & $7.947 \mathrm{E}-2$ & 642 & 104.6 & $1.38(-1)$ & 758 \\
\hline 65536 & 204 & 0.3452 & 592 & 418.5 & 0.502 & 834 \\
\hline 131072 & 817 & 1.361 & 600 & 1674 & 1.986 & 843 \\
\hline 262144 & 3268 & 5.361 & 609.5 & 6696 & 7.892 & 848 \\
\hline 524288 & 13070 & 21.345 & 612 & 26783 & 31.41 & 853 \\
\hline 1048576 & 52281 & 85.205 & 614 & $1.071 E 5$ & 125.36 & 855 \\
\hline
\end{tabular}

Table 1 shows some comparative performance results for solution of the same benchmark problem $(N$ random sources of random intensities $q \in(0,1)$, and $M=N+1$ random receivers) using a serial CPU programming and the GPU (everything in single precision). Large times given in italic are estimates of the CPU time based on $N^{2}$ scaling. It is important to note that we used no optimizations afforded by our Intel CPU (SSE extensions and parallelization), and the results for the CPU are not meant to indicate the best CPU 
performance achievable. Rather, our goal was to compare with the simplest baseline case on the CPU. The same applies to all other comparisons with CPU results in the subsequent sections. While in a sense this is unfair to the CPU implementations, our goal is not to perform a "competition" between the two architectures, but rather use the straightforward CPU implementation to guide GPU code development.

The table and the associated graph for the potential calculation (see 6) show that the timing of the CPU is consistent with the theoretical dependence, Time $=A N^{2}$, while the GPU timing approaches quadratic dependence in an asymptotic way. This is also clear from the time ratio between the CPU and GPU processes shown in the table, which stabilizes at relatively large values of $N$.

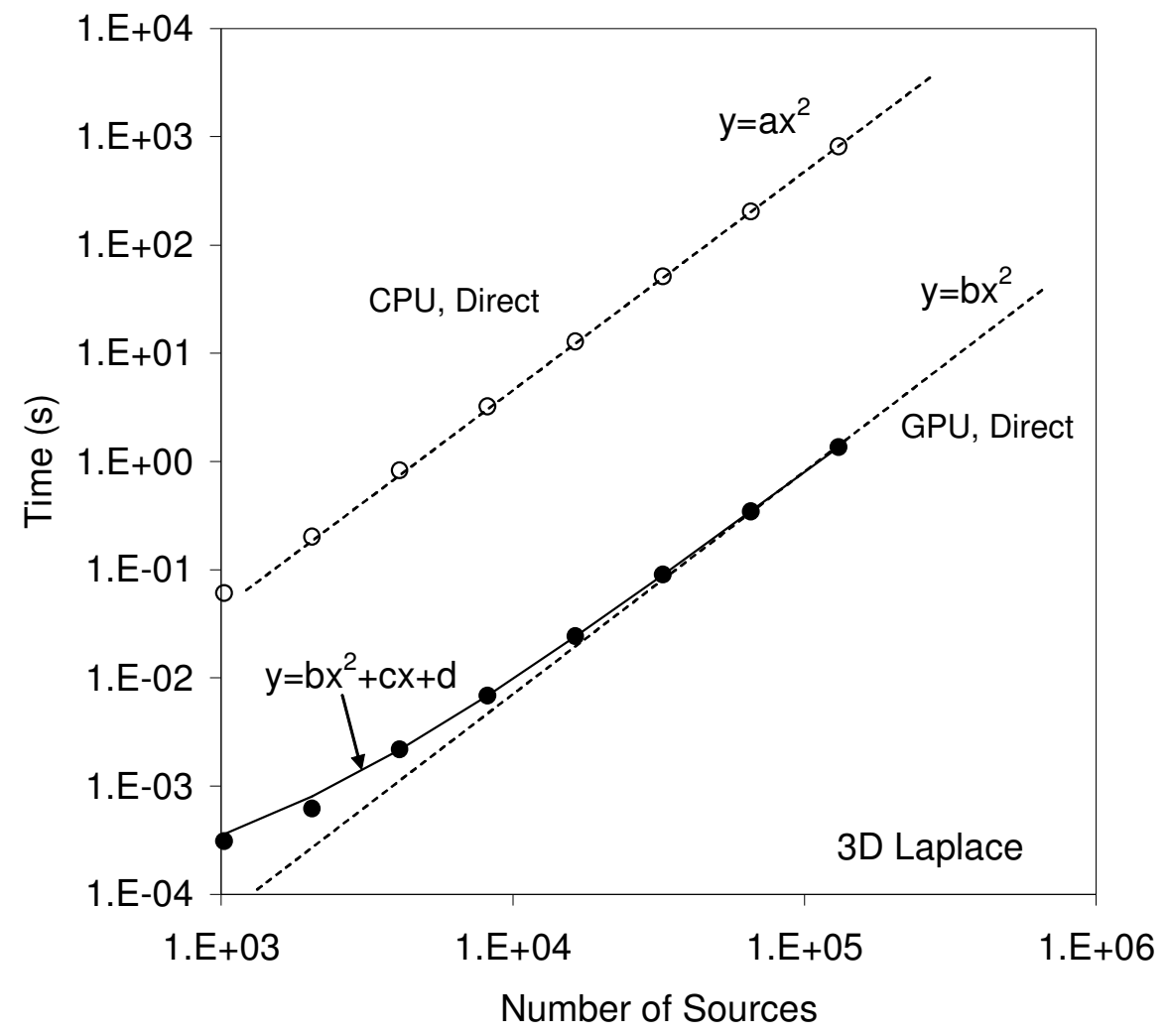

Figure 6: Comparison of the time required for solution of the same problem (direct summation (1)) using a serial CPU code and the GPU. $N$ sources and $M=N+1$ receivers are unstructured sets of random data.

This result may be explained by analyzing the algorithm. Indeed, a full GPU load is achieved only when the number of blocks exceeds 32. Taking into account that in our case each block contains 256 receivers this results in the size of the receiver set $M \geqslant 8192$ for a full load. Second, as mentioned earlier, there exists an overhead in the computations related to read/write operations from main memory. This overhead is proportional to the number of sources and receivers. In other words, the read/write time scales linearly with $N$. Third, some time that is much larger than the time needed for a single arithmetic operation is needed to access the first pointer in the array of receivers, sources, and the output vector. Assuming that a coalesced reading/writing is realized, we should simply add some constant to the execution time. With this reasoning the model of GPU timing for direct summation can be written as

$$
\text { Time }=A N^{2}+B N+C,
$$

where $A, B$, and $C$ are some constants. Assuming that this model applies for $N \geqslant 8192$ we took three 
values from the table at $N=8192,32768$, and 131072 and determined these constants. The resulting fit line is shown in Fig. 6. It is seen that this fit can be continued even below 8192 and is close enough to the observed time.

\subsection{Sparse matrix vector multiplication}

The sparse matrix vector multiplication step that computes the local interactions is one of the most time consuming parts of the FMM. Moreover, the performance achieved for this part can be applied to acceleration of the entire FMM algorithm by proper selection of the maximum level of the octree space subdivision, which controls the amount of the interactions computed directly and via the FMM approximation. Our local interaction algorithm is in many aspects similar to the total direct summation algorithm described above, but with several necessary modifications, dictated by the FMM structure. The impact of these modifications on the algorithm performance is considered below.

Let us consider some receiver box $b_{r}$ at level $l_{\max }$ containing $r$ receivers. The neighborhood of this box, if it is not located on the domain boundary consists of at most 27 non-empty source boxes, $b_{s}$. Assume, for simplicity, that each of these boxes contain approximately $s$ sources. Therefore, to obtain the required $r$ sums for box $b_{r}$ we need to perform multiplication of a matrix of size at most $r \times 27 s$ by a vector of length at most $27 s$.

The hierarchical spatial ordering based on bit-interleaving that we use provides that all receivers and sources in a given box are allocated in the memory contiguously. In other words, if we have a list of sources, then to access data for a given box $b_{s}$ we just need to pass a pointer corresponding to box $b_{s}$ and the amount of data in the box. Instead of this, we can also use a bookmark list that provides the minimum and maximum source indices for a given box, where all the source indices are stored in long array in global memory. The same remark applies to the receiver boxes.

Since the neighbor relation is not hierarchical (like the child-parent relationship), an additional data structure that lists all the source box neighbors for a given receiver box (our algorithm is adaptive, and, in fact the number of occupied source boxes in the neighborhood can be an arbitrary number from 0 to 27). Data for up to $27 s$ sources required for one receiver box execution are allocated in the memory not in subsequent way, but by pieces, each of which needs a pointer.

The main modifications of the execution model on the GPU then appear to be as follows.

1. Each block of threads executes computations for one receiver box (Block-per-box parallelization).

2. Each block of threads accesses the data structure.

3. Loop over all sources contributing to the result requires partially noncoalesced read of the source data (box by box).

These features substantially slow down the performance. Indeed due to these modifications not all the threads in the block are employed, or they may be employed unequally (the number of receivers varies from box to box). Having the receiver box executed by a block is dictated by the necessity to put source data required for processing of this block into the shared memory and employ as many threads as possible for the data read operation. Also, only receivers of the same box share the same set of sources. As mentioned earlier, due to the non-hierarchical nature of the neighbor relation, initial access to any box should be considered as a random data access, and these reads each pay the corresponding initial access penalty.

\subsubsection{Model for run times of the sparse matrix vector product}

We use actual run times for the sparse matrix vector multiplier FMM evaluation of (1) to develop a theoretical performance model. We first consider the performance for the values of the clustering parameter $s$, or $l_{\max }$ 
that are optimal for the CPU codes ( $p=8$ and $p=12$ for which we set data structures with the requirement $s \leqslant s_{\max }=60-80$ ). At optimality the sparse and dense matrix vector costs balance, though since the level changes discretely over integers, in practice the balance is approximate. Fig. 7 shows the performance for this case.

Two things can be noticed immediately. First, GPU acceleration of the FMM compared to the CPU is very nonuniform. It varies by an order of magnitude for the same $l_{\max }$. On the other hand, it is not difficult to see that the GPU timing for a given $l_{\max }$ changes slowly with increase of data size. In theory, the complexity of the matrix vector product for fixed $l_{\max }$ should grow quadratically with $N$, which is perfectly reflected in the CPU timing. The "nonuniform" acceleration in this case is because on the CPU performance is determined by the data size, while on the GPU it is determined by the depth of the octree $\left(l_{\max }\right)$.

Eq. (26, right) provides the time for the CPU implementation, where the cost of data access is assumed negligible. This dependence shows that at fixed $l_{\max }$ the time is proportional to $N^{2}$, which is in very good agreement with the experimental results. However, in the GPU realization the time appears to be a different function of $N^{2}$. It is more or less clear that this is due to the substantial costs of data access. Since the algorithm is executed box by box, where the number of boxes is a function of $l_{\max }$ the number of random access operations is also is a function of $l_{\max }$. The number of boxes is $\sim 8^{l_{\max }}$, or $N / s$ In this case we can evaluate the GPU time as follows.

$$
\text { Time }=A N s+B \frac{N}{s}+C N, \quad s=N \cdot 8^{-l_{\max }} .
$$

The first term represents the number of arithmetic operations and similar to (26, right). The second term represents the number of boxes and characterizes the time for random memory access. The last term is the cost of reading/writing of data sets of length $N$ in a sequential manner or for fast access to the shared memory or other coalesced read/write. Model constants can be evaluated from three experimental data points and the fit is plotted in figure Fig. 7. Note that for this fit we determined the constants only from three data points for level $l_{\max }=5$. However, the fit works nicely for the maximum subdivision levels 4 and 3 as well. Some deviation is observed for level 2, which may be explained by the fact that for these levels the data size becomes too small to provide a good GPU load.

The nonnegligible magnitude of the second term in the right hand side of Eq. (31) has far-reaching consequences. Indeed, if Eq. (26, right) shows that to reduce the time for this FMM step for fixed $N$ one should decrease $s$ (or increase $l_{\max }$ ) as much as possible, then Eq. (31) claims that substantial decreasing or increasing of $s$ will increase the computation time, and there exists a unique minimum of function Time $(s)$. This minimum is realized at $s=s_{\text {opt }}^{(\text {sparse })}$ and the optimum time is $\operatorname{Time}_{o p t}^{(\text {sparse })}=\operatorname{Time}\left(s_{o p t}^{(\text {sparse })}\right)$, which can be expressed in terms of the hardware and algorithm related constants $A, B$, and $C$ as

$$
s_{\text {opt }}^{(\text {sparse })}=\left(\frac{B}{A}\right)^{1 / 2}, \quad \operatorname{Time}_{\text {opt }}^{(\text {sparse })}=\left[2(A B)^{1 / 2}+C\right] N .
$$

The latter equation also shows that the optimal time for the algorithm is linear in $N$.

The estimate of fitting constants, which we obtained shows that $s_{\text {opt }}^{\text {(sparse })}=91$, which is larger than the size of the maximum cluster used for the above computations. We note then that the octree is a discrete object and the number of levels is an integer and if we build any continuous model approximation and find the optimum, this optimum in general cannot be achieved exactly. So the only way to change the performance for a given $N$ is to change $l_{\max }$ (or sufficiently increase $s_{\max }$ ). To check these predictions we performed a run for larger $s_{\max }(=320)$ to see how the performance changes.

Figure 8 demonstrates one order of magnitude of increase of the relative GPU performance. Indeed the CPU timing is proportional to the number of arithmetic operations. For large clusters, of course, this number increases. However the GPU time decreases (compare with Fig. 7). This decrease is not huge, but the density 


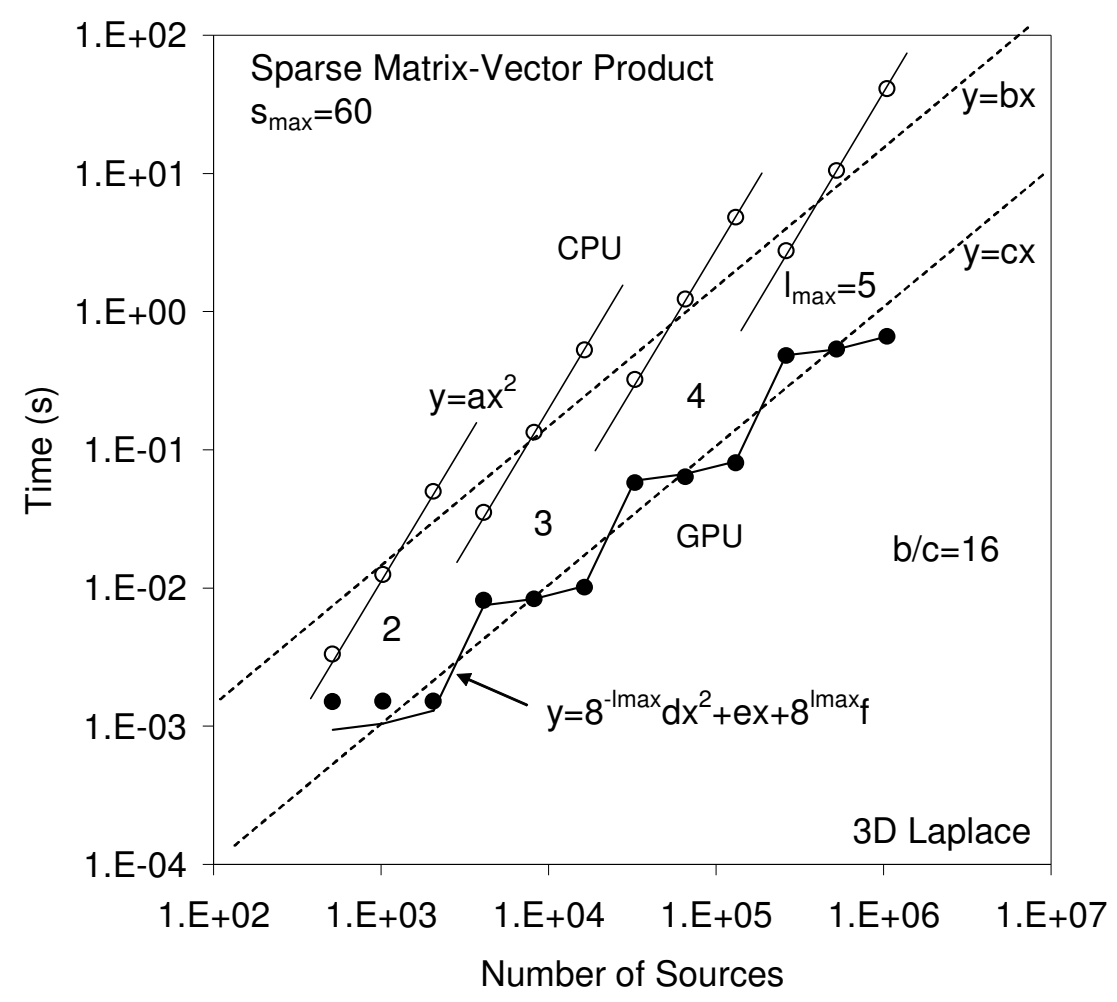

Figure 7: Performance of sparse matrix-vector multiplier used in the FMM for maximum cluster parameter $s_{\max }=60$. The numbers $2, \ldots 5$ near the curves show the maximum level of octree space subdivision $l_{\max }$. Solid and dashed lines show different data fits.

of arithmetic operations increases and the Gflops count increases at least by one order of magnitude. One can treat this as an amortization of the random global memory access cost, or simply look at formula (31).

It is seen that the qualitative behavior of the CPU time remains the same and is well described by Eq. (26, right). The qualitative behavior of the GPU time however changes, and the step function of Fig. 7 turns into almost linear dependence on $N$. (It is interesting to note that the cost model for the GPU sparse matrix product is qualitatively similar to the cost model for the entire FMM - compare Eqs (27) and (31)). We also plotted the fit (31) with the same $A, B$, and $C$ as for Fig. 7. It is clear that it is consistent with the large cluster case as well. Also the closeness of the GPU time to the linear dependence in this case shows that the selection of the cluster size is close to optimal (for optimal size the dependence is linear, see Eq. (32)). Further increase of the cluster size ( $l_{\max }$ reduction) is not beneficial for performance. The GPU dependence becomes qualitatively similar to the CPU dependence and the execution time increases (see Eq. (31)). It is also interesting that cluster sizes, which we consider as optimal $(\sim 256)$ are also optimal for the thread block size of the GPU. Also the maximum number threads in the block on the processor we used is limited by 512, for which the performance of the GPU downgrades, and the imbalance between the random global memory access costs and arithmetic complexity becomes substantial.

We note then that for a given algorithm for sparse matrix vector multiplication and hardware, which determine the constants $A, B$, and $C$ in Eq. (32) we have a minimum possible time for the entire FMM. Indeed any other FMM procedure can only increase the total time, and in any case it will be larger than Time ${ }_{\text {opt }}^{\text {(sparse) }}$ given by Eq. (32).

Because of the different complexity dependences for the CPU and GPU, to be fair, we should rather 


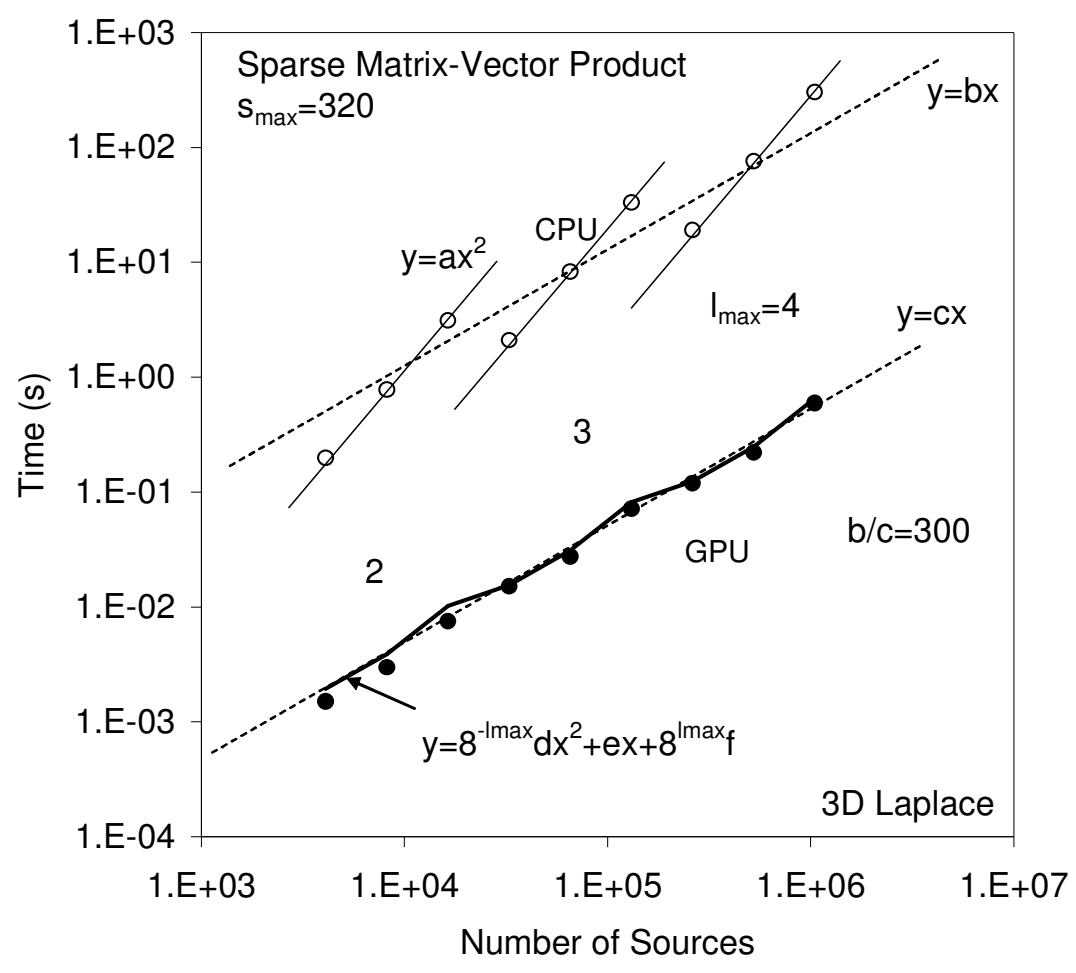

Figure 8: The same as Fig. 7, but for $s_{\max }=320$.

compare the best performing cases for each type of architecture. Table 2 shows the time ratio for the best performing cases ( $p=8$ and 12, potential computations only). Again, as mentioned previously, the CPU times are for a serial implementation that does not take advantage of architecture specific vectorization instructions available, such as SSE.

\begin{tabular}{|l|l|l|l|}
\hline \multicolumn{4}{|c|}{ Table 2. Sparse m/v mult. at best settings } \\
\hline$N$ & CPU(s) & GPU(s) & Ratio \\
\hline 32768 & 0.322 & 0.0151 & 21 \\
\hline 65536 & 1.23 & 0.0275 & 45 \\
\hline 131072 & 4.81 & 0.0713 & 68 \\
\hline 262144 & 2.75 & 0.120 & 23 \\
\hline 524288 & 10.5 & 0.221 & 47 \\
\hline 1048576 & 41.0 & 0.596 & 69 \\
\hline
\end{tabular}

\subsection{Other FMM subroutines}

Below we briefly describe each routine performing the other steps of the FMM and provide some data on its performance.

\subsubsection{Multipole expansion generator}

This subroutine generates $S$ expansions for each source box at the finest level, which is Step 1) of the FMM Upward Pass. As mentioned above, this procedure is equivalent to generation of the $R$ basis for each source 
in the given box followed by consolidation of all expansions. The $R$ basis is generated by the recursions (15) and can be performed for each source independently. So one solution for parallelization is to assign each thread to handle one source expansion.

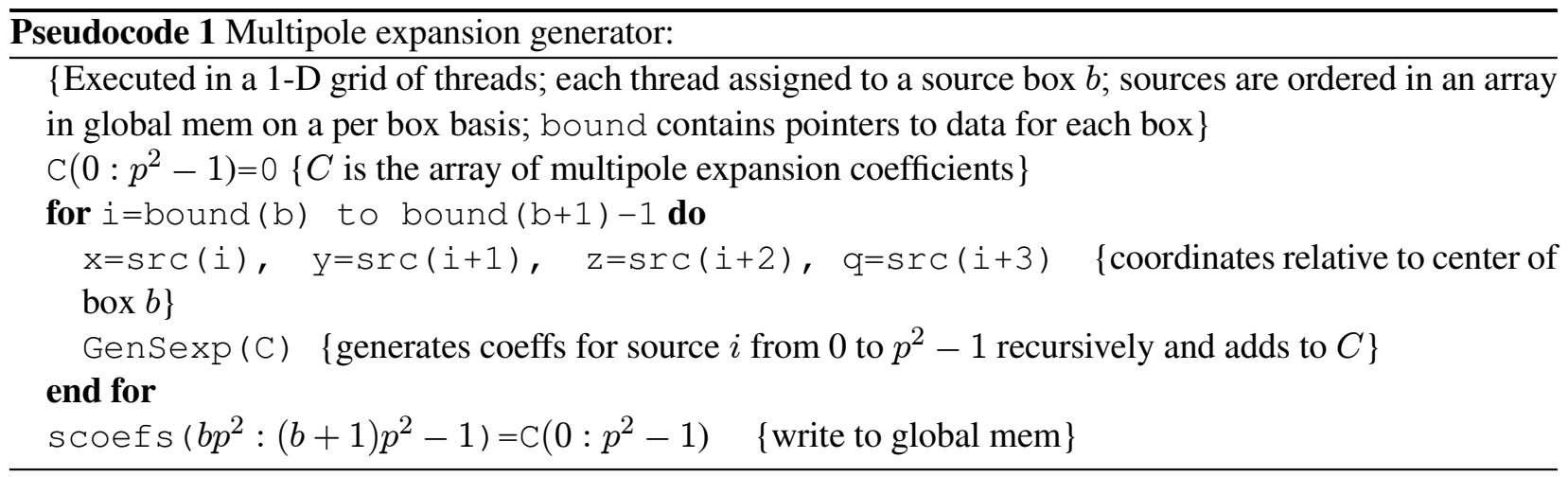

A drawback of this method is that after generation of expansions they need to be consolidated, which will necessitate data transfer to GPU global memory, unless they form a block of threads handled by one processor. The block size for execution of any subroutine in GPU can be defined by the user, but it is fixed during execution. In the FMM each box may have different number of sources. Thus if a source box is handled by a block of threads then threads could be idle, which, of course, reduces the utilization efficiency. GPU accelerations compared to the serial CPU code in this case are in range 3-7, which appear to be rather low compared with performance of other routines.

The efficiency of the $S$ expansion generator substantially increases with a different parallelization model: one thread per box. In this case one thread performs expansion for each of $s$ sources in the box and consolidates these expansion. So one thread produces full $S$ expansion for the entire box. The advantage of this approach is that the work of each thread is completely independent and so there is no need for shared memory. This perfectly fits the situation when each box may have different number of sources, as the thread that finishes work for a given box simply takes care of another box, without waiting or need for synchronization with other threads. The disadvantage of this approach is that to realize the full GPU load the number of boxes should be sufficiently large. Indeed, if an optimal thread block size is 256 and there are 16 multiprocessors (so we need at least 32 blocks of threads to realize an optimal GPU load), then the number of boxes should be at least 8192 for a good performance. Note that $l_{\max }=4$ we have at most $8^{4}=4096$ boxes, and for $l_{\max }=5$ this number becomes $8^{5}=32768$ boxes. So the method can work efficiently only for large enough problems.

\begin{tabular}{|l|l|l|l|l|l|l|}
\hline \multicolumn{7}{|l|}{ Table 3. Performance of $S$ expansion generator $(p=12)$} \\
\hline & $s_{\max }=60$ & \multicolumn{3}{l|}{$s_{\max }=320$} \\
\hline$N$ & CPU(s) & GPU(s) & Ratio & CPU(s) & GPU(s) & Ratio \\
\hline 16384 & 0.0281 & $4.22 \mathrm{E}-03$ & 6.7 & $2.78 \mathrm{E}-02$ & $2.34 \mathrm{E}-02$ & 1.2 \\
\hline 131072 & 0.229 & $5.93 \mathrm{E}-03$ & 38.6 & $2.23 \mathrm{E}-01$ & $2.52 \mathrm{E}-02$ & 8.9 \\
\hline 262144 & 0.496 & $1.73 \mathrm{E}-02$ & 28.6 & $4.53 \mathrm{E}-01$ & $1.05 \mathrm{E}-02$ & 43.3 \\
\hline 1048576 & 1.92 & $4.61 \mathrm{E}-02$ & 41.6 & $1.81 \mathrm{E}+00$ & $3.81 \mathrm{E}-02$ & 47.4 \\
\hline
\end{tabular}

Results for $p=12$ are presented in Table 3. The performance on the CPU does not depend on the number of boxes and is a linear function of $N$. On the other hand the performance of GPU in this range of $N$ and cluster sizes is heavily influenced by the number of boxes, or $l_{\max }$. This fact is due to the algorithm we used, and as we explained above a full GPU load can be achieved by this algorithm only at levels $l_{\max } \geqslant 4$. For 
$s_{\max }=320$ criterion $l_{\max } \geqslant 4$ is achieved only for $N \geqslant 262144$ and after that one can expect more or less linear dependence of the GPU time on $N$.

Of course, there are further opportunities to improve performance for $l_{\max }<4$. However, the time spent for the $S$ expansion generation usually does not exceed a couple of percent of the overall FMM run time and also the FMM on the GPU is efficient only for relatively large problems.

\subsubsection{Local expansion evaluator}

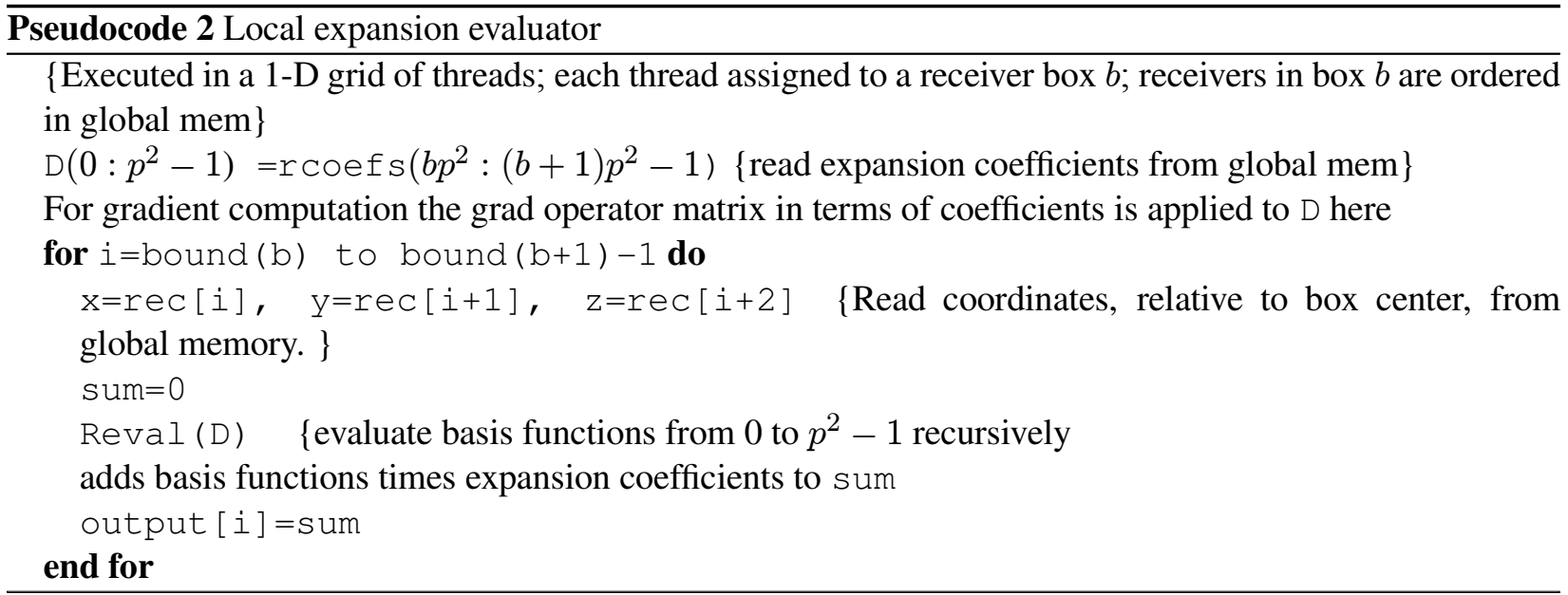

This subroutine is part of Step 1 of the final summation. The reason why it is described out of order is that it is very similar to the $S$ expansion generator discussed above. For parallelization of this routine the one thread per box strategy is used. The performance of this subroutine is approximately the same as of the $S$ expansion generator. Finally we mention that both $S$ and $R$ subroutines described above use fast (diagonal) renormalization of the expansion, which provides more compact storage of the rotation operators.

\subsubsection{LevelUp}

The subroutine LevelUp is the core of the Step 2 in the FMM Upward Pass. It takes as input $S$ expansions for all source boxes at level $l$ and produces expansions for all source boxes at level $l-1$. Being called in a loop for $l=l_{\max }, \ldots, 3$ it completes the full Upward Pass of the FMM. The kernel of routine LevelUp consists of the $S \mid S$ translator, which takes as input an $S$ expansion from one box (usually the child) and produces a new (translated) $S$ expansion at the center of another box (the parent). To produce the $S$ expansion for the parent box all $S \mid S$ translated expansions from its children boxes should be consolidated.

We have not yet come upon an optimal strategy for this subroutine. The current version is based on the fact that the resulting $S$ expansions for the parent boxes can be generated independently. So each thread can take care on one parent box. However, the work load of the GPU in this case becomes very small for low $l_{\max }$ and more or less reasonable speedup can be achieved only if several threads are allocated to process a parent box. Since each parent box in the octree has at most 8 children and for each child $S \mid S$ translation can be performed independently, we used two dimensional $32 \times 8$ blocks of threads and one dimensional grid of blocks. In this setting each parent box was served by 8 threads, with the thread id in $y$ varying from 0 to 7 for identification of the child boxes.

As mentioned above we use the RCR decomposition of translation operators. In fact each rotation is decomposed further into $\alpha$ and $\beta$ rotations, corresponding to the respective Euler angles. So the translation 


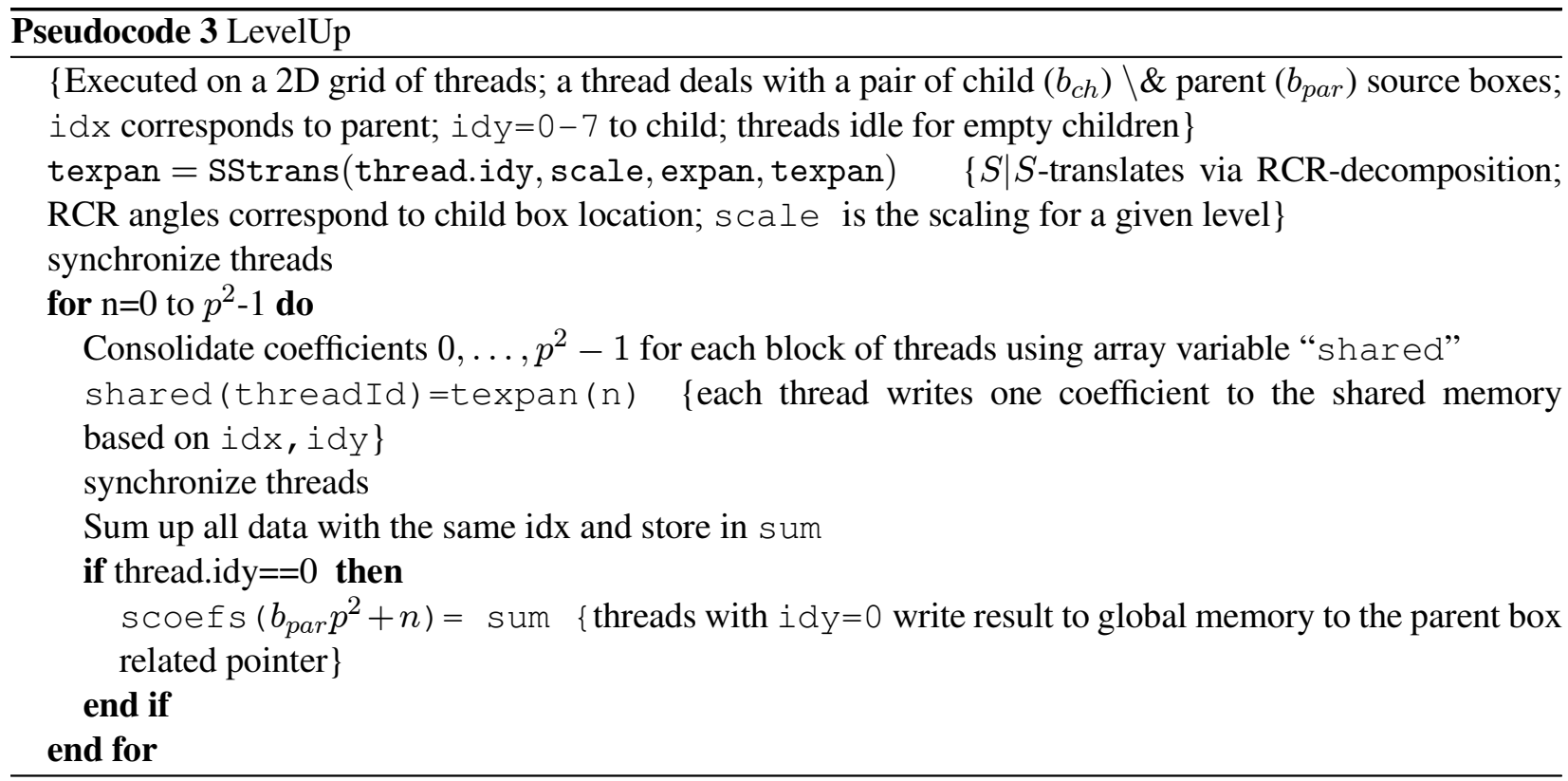

matrix $(\mathbf{S} \mid \mathbf{S})(\mathbf{t})$ in our case is decomposed as

$$
(\mathbf{S} \mid \mathbf{S})(\mathbf{t})=\mathbf{A}^{-1}(\alpha) \mathbf{B}(\beta) \underline{(\mathbf{S} \mid \mathbf{S})}(t) \mathbf{B}(\beta) \mathbf{A}(\alpha),
$$

where $(t, \beta, \alpha)$ are the spherical coordinates of the translation vector $\mathbf{t}$, while $\mathbf{A}$ and $\mathbf{B}$ are the rotation matrices and $(\mathbf{S} \mid \mathbf{S})$ is the coaxial translation matrix (see also Fig. 9). A peculiarity of the $S \mid S$ translation is that all translations for a given level have the same length $t$, there are only two different angles $\beta$, and four different $\alpha$ (in Fig. 9 only one translation is shown, while 7 other translations have symmetric properties). In addition there is a central symmetry of the translation operator:

$$
(\mathbf{S} \mid \mathbf{S})(-\mathbf{t})=\mathbf{A}^{-1}(\alpha) \mathbf{F B}(\pi-\beta) \underline{(\mathbf{S} \mid \mathbf{S})}(t) \mathbf{B}(\pi-\beta) \mathbf{F A}(\alpha),
$$

where $\mathbf{F}$ is a very simple constant diagonal flip operator. This allows to use only one constant matrix $\mathbf{B}(\pi / 4)$ for all $S \mid S$ translations. We use these facts for data compression, which allows us to put dense constant matrices $(\mathbf{S} \mid \mathbf{S})(t)$ and $\mathbf{B}(\pi / 4)$ into the fast (constant or shared ) memory. The operator $\mathbf{A}(\alpha)$ is diagonal and the only input needed for its computation is the angle $\alpha$, so there is no cost to read or write this operator to global memory.

Operations $\mathbf{A}(\alpha) \phi, \mathbf{B}(\beta) \phi, \mathbf{F} \phi$, and $(\mathbf{S} \mid \mathbf{S})(t) \phi$ are implemented as static inline functions in CUDA extended $\mathrm{C}$ and compiled with the kernel library. Each thread calls these inline functions after it reads the input data from the global GPU memory. After performing the $S \mid S$ translation the threads are synchronized, and a reduction operation is used to consolidate the obtained $S$ expansions and write them back to the global memory.

\begin{tabular}{|l|l|l|l|l|l|l|l|l|l|}
\hline \multicolumn{2}{|c|}{ Table 4. Performance for Step 2 of the FMM Upward Pass } \\
\hline & $p=4$ & \multicolumn{1}{|c|}{$p=8$} & \multicolumn{1}{l|}{} & \multicolumn{1}{l|}{} \\
\hline$l_{\max }$ & CPU(s) & GPU(s) & Ratio & CPU(s) & GPU(s) & Ratio & CPU(s) & GPU(s) & Ratio \\
\hline 3 & $3.07 \mathrm{E}-04$ & $1.44 \mathrm{E}-04$ & 2.1 & $1.87 \mathrm{E}-03$ & $9.26 \mathrm{E}-04$ & 2.0 & $4.68 \mathrm{E}-03$ & $2.49 \mathrm{E}-03$ & 1.9 \\
\hline 4 & $2.97 \mathrm{E}-03$ & $4.57 \mathrm{E}-04$ & 6.5 & $1.61 \mathrm{E}-02$ & $2.18 \mathrm{E}-03$ & 7.4 & $4.26 \mathrm{E}-02$ & $5.62 \mathrm{E}-03$ & 7.6 \\
\hline 5 & $2.39 \mathrm{E}-02$ & $2.34 \mathrm{E}-03$ & 10.2 & $1.29 \mathrm{E}-01$ & $1.16 \mathrm{E}-02$ & 11.1 & $3.45 \mathrm{E}-01$ & $3.09 \mathrm{E}-02$ & 11.2 \\
\hline 6 & $1.86 \mathrm{E}-01$ & $1.89 \mathrm{E}-02$ & 9.9 & $1.02 \mathrm{E}+00$ & $9.39 \mathrm{E}-02$ & 10.9 & $2.72 \mathrm{E}+00$ & $2.55 \mathrm{E}-01$ & 10.6 \\
\hline
\end{tabular}

Table 4 shows performance of the CPU and GPU subroutines to perform entire Step 2 of the Upward Pass (so the LevelUp is called $l_{\max }-2$ times). The execution time here is presented as a function of $l_{\max }$. 


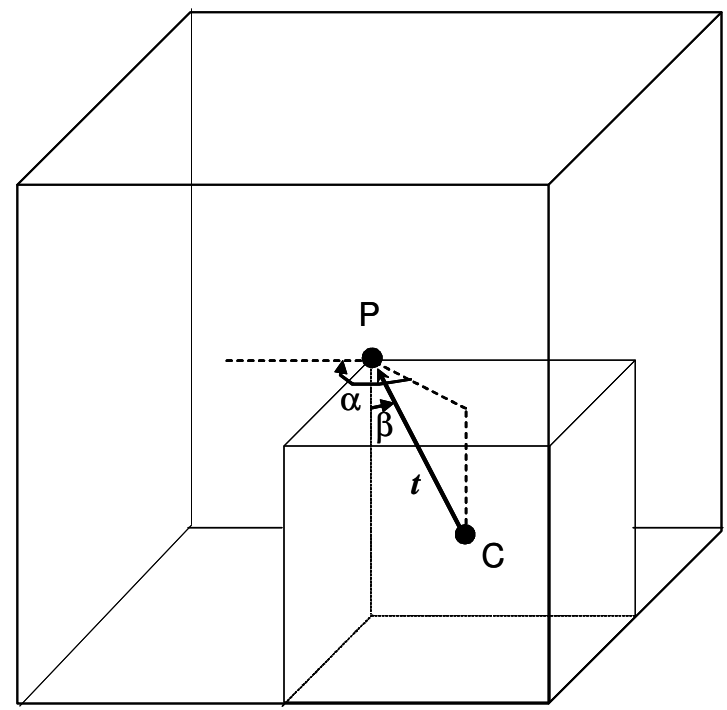

Figure 9: Translation from the center of a child box (C) to its parent (P). $\alpha$ and $\beta$ are Euler rotation angles and $\mathbf{t}$ is the translation vector.

Indeed, the complexity of this step depends only on the number of source boxes, not on $N$. This number for uniform distributions is approximately $8^{l}$ for level $l$, and this value is used a benchmark .

The obtained speedups are in range 2-11, where 2 corresponds to $l_{\max }=3$ which is only 512 children and 64 parent boxes at most. So the efficiency of translation/per thread parallelization is low (as we mentioned for the current architecture sizes involving 8192 parallel processes or more can be run at full efficiency. In fact, even $l_{\max }=4$ appears to be a bit low for a full load. For levels $l_{\max } \geqslant 5$, the GPU speed up over the serial code is approximately one order of magnitude, which includes computations not only for $l_{\max }$, but for all levels from $l_{\max }$ to 3 . Note that the speedups are uniform for all $p$ considered.

Despite the speedups for this step appear to be lower than for other steps presented above, two observations make the obtained results satisfactory for the entire FMM.

The first observation is that, as will be seen below, the FMM on the GPU is optimal for a smaller $l_{\max }$ than on the CPU. The optimization study for the sparse matrix vector multiplier clearly indicates that for the benchmark case $l_{\max }$ does not exceed 4 for problem sizes $N \leqslant 2^{20}$. In most cases $l_{\max }$ for the GPU is equal to $l_{\max }-1$ for CPU, while for case $p=4$ it may be even smaller (4 instead of 6). Since the optimal settings for the CPU or GPU are not optimal for each other we can create a table for the best performing cases on each architecture and compare speedups as we did before for the sparse matrix vector multiplier (Table 5). The value $\infty$ here appears due to Step 2 of the Upward Pass is not needed for $l_{\max }=2$ (or its execution time is 0 ). We also put N/A for the cases which are not optimal. The speedups then appear to be in the same range as for other routines.

Table 5. Effective Performance (the best settings for CPU and GPU) for Step 2 of the FMM Upward Pass

\begin{tabular}{|l|l|l|l|l|l|l|l|l|l|l|}
\hline \multicolumn{2}{|l|}{$l_{\max }$} & \multicolumn{3}{l|}{$p=4$} & \multicolumn{2}{l|}{$p=8$} & \multicolumn{2}{l|}{$p=12$} \\
\hline CPU & GPU & CPU(s) & GPU(s) & Ratio & CPU(s) & GPU(s) & Ratio & CPU(s) & GPU(s) & Ratio \\
\hline 3 & 2 & $3.07 \mathrm{E}-04$ & $0.00 \mathrm{E}+00$ & $\infty$ & $1.87 \mathrm{E}-03$ & $0.00 \mathrm{E}+00$ & $\infty$ & $4.68 \mathrm{E}-03$ & $0.00 \mathrm{E}+00$ & $\infty$ \\
\hline 4 & 3 & $2.97 \mathrm{E}-03$ & $1.44 \mathrm{E}-04$ & 21 & $1.61 \mathrm{E}-02$ & $9.26 \mathrm{E}-04$ & 17 & $4.26 \mathrm{E}-02$ & $2.49 \mathrm{E}-03$ & 17 \\
\hline 5 & 4 & $2.39 \mathrm{E}-02$ & $4.57 \mathrm{E}-04$ & 52 & $1.29 \mathrm{E}-01$ & $2.18 \mathrm{E}-03$ & 59 & $3.45 \mathrm{E}-01$ & $5.62 \mathrm{E}-03$ & 61 \\
\hline 6 & 4 & $1.86 \mathrm{E}-01$ & $4.57 \mathrm{E}-04$ & 408 & N/A & N/A & N/A & N/A & N/A & N/A \\
\hline
\end{tabular}

The second observation is that the Upward Pass is a very cheap step of the FMM and normally takes not 
more than $1 \%$ of the total time. This also diminishes the value of investing substantial resources in achieving high speedups for this step.

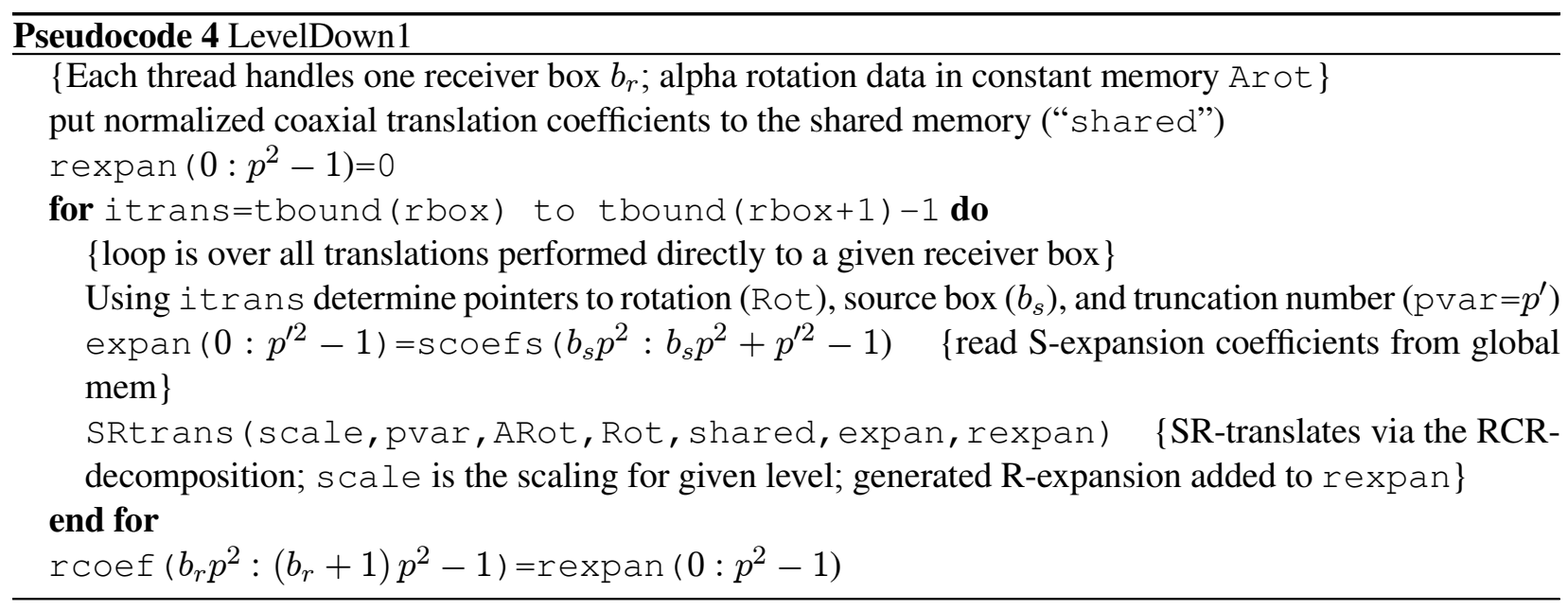

\subsubsection{LevelDown}

Perhaps this is the most challenging subroutine for efficient parallelization. It generates $R$-expansions for all receiver boxes at level $l$ using as input data ( $S$ expansions) from source boxes of the same level and $R$ expansions from level $l-1$. Despite the fact that this subroutine is thought of as a single entity, in our code it is actually comprised of three global device functions, LevelDown1, LevelDown2, and LevelDown3, which correspond to the stencil translation scheme. LevelDown 1 performs all required multipole-to-local $(S \mid R)$ translations from the source boxes to the receiver boxes of the same level (white boxes in Fig. 4), followed by consolidation. LevelDown 2 performs all necessary $S \mid R$ translations from the other subset of the source boxes to the parent receiver box (gray boxes in Fig. 4), followed by consolidation. LevelDown3 performs the local-to-local $(R \mid R)$ translations from the parent box to its children and adds the result to that computed by LevelDown 1 .

The LevelDown 3 subroutine is very similar to LevelUp, since it performs $R \mid R$ translations from parent box to its 8 children. So we applied similar tricks to reduce the amount of translation data for this part and use fast access memory to store these data. One can even use the tables and data for the LevelUp to estimate the costs and speedups for LevelDown3.

Efficient parallelization is more difficult for LevelDown 1 and LevelDown2. We tried several translation schemes that demonstrated that the speedups achieved on these routines is only in the range 2-5 and we still are looking for better solutions. Our current version of these subroutines work as follows.

LevelDown 1 assigns a single receiver box at level $l$ to a single thread. In this case thread synchronization is not needed and the nonuniformity of the receiver box neighborhoods is not very important. This thread performs up to $109 S \mid R$ translations according to the stencil structure, consolidates all expansions, and writes the result to the global memory. LevelDown 2 parallelized differently, due to the number of parent boxes can be substantially small. In this subroutine a block of threads performs all operations for a single parent box. Each thread is responsible for one $S \mid R$ translation. The maximum number of the $S \mid R$ translations to the parent receiver box in the stencil is 80 . So up to 80 threads are employed at a time. This number is a bit low for a good performance of the GPU, but allows to all threads perform read/write operations efficiently and use the shared memory.

For the $S \mid R$ translation we use a decomposition similar to (33) (one should change there $S \mid S$ to $S \mid R$ ). The difference is that for the $S \mid R$ translations we need much more translation data, which can fit neither 


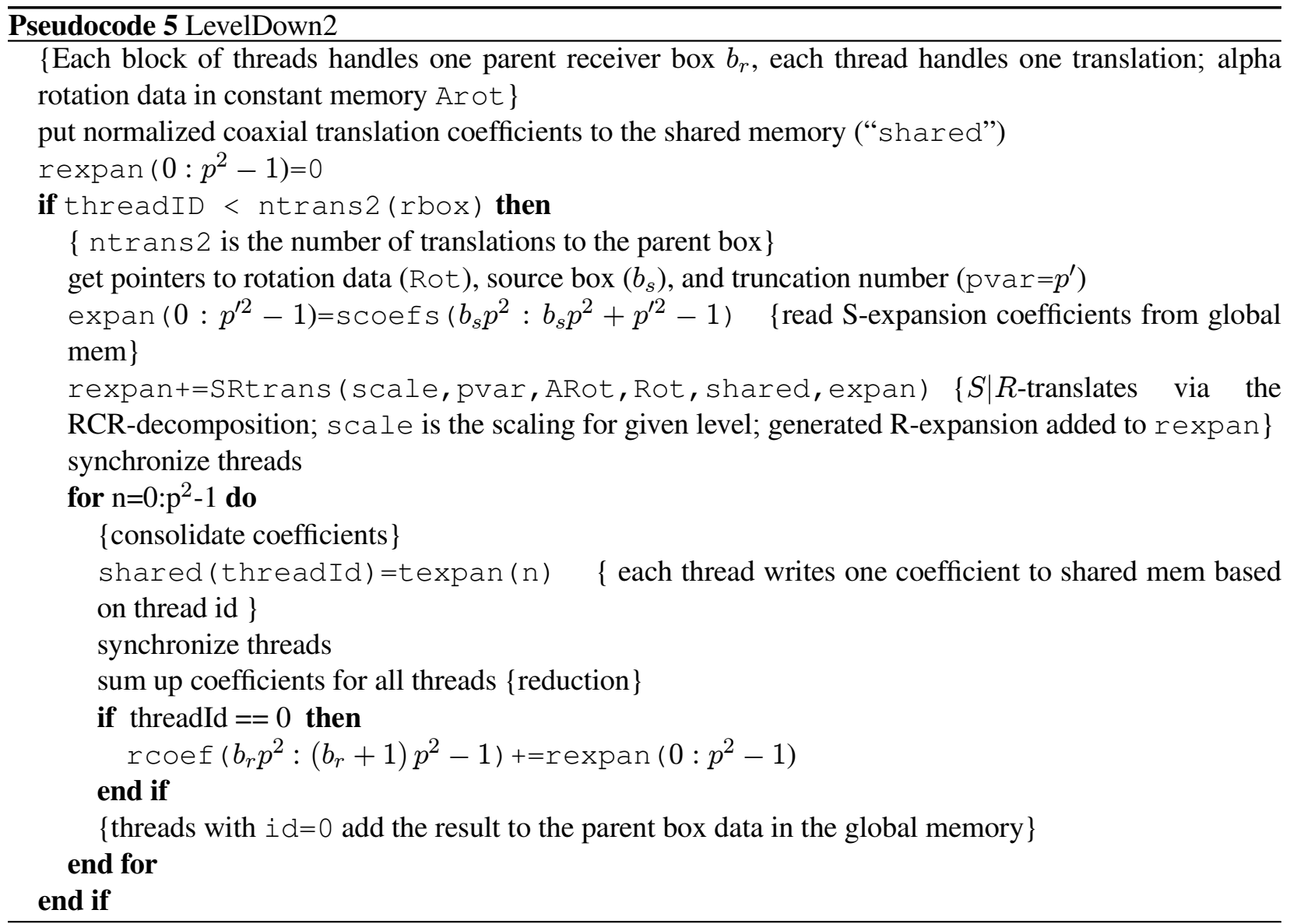

in shared nor in constant memory. (This fact is an indication that research on more compact translation operator decompositions of the same or better complexity is necessary. Ideas for some such schemes are discussed in [11] and, even if they are not the best for CPU implementations, in future research we are going to try to implement them on the GPU). Indeed, in the stencil there are 48 different values of the angle $\alpha, 49$ different values of the angle $\beta$, and 14 different translation distances. To reduce the amount of translation data read from the global memory, all $\alpha$ 's and translation distances are put in constant memory. Only one $(S \mid R)$ coaxial translation matrix needs to be loaded in addition, and it is rescaled each time to produce actual coaxial operator. This is based on use of the decomposition

$$
\underline{(\mathbf{S} \mid \mathbf{R})}(t)=\frac{1}{t} \Lambda\left(\frac{1}{t}\right) \underline{(\mathbf{S} \mid \mathbf{R})}(1) \Lambda\left(\frac{1}{t}\right),
$$

where $\Lambda$ is a diagonal matrix whose $n$th subspaces has $1 / t^{n}$ normalization. In this case $(\mathbf{S} \mid \mathbf{R})(1)$ easily fits shared or constant memory.

More storage related problems are presented by the operator $\mathbf{B}(\beta)$. In principle, it can also be decomposed into the product of two constant matrices that cause an axis flip, and a diagonal matrix. However, tests on the CPU showed that this method is not efficient on the CPU and we did not proceed in this way. This operator is a block dense matrix (dense for each rotation subspace) and its size for $p=12$ is 1152 floats. To store 49 different operators we need about $226 \mathrm{kB}$ of memory. An ideal solution would be to put these data to the constant memory, but it is limited to about $60 \mathrm{kB}$ for the NVIDIA 8800 GTX GPU we use. If hardware with sufficient fast access memory becomes available, the algorithm can be modified, and we expect sensible and more usual GPU accelerations. 


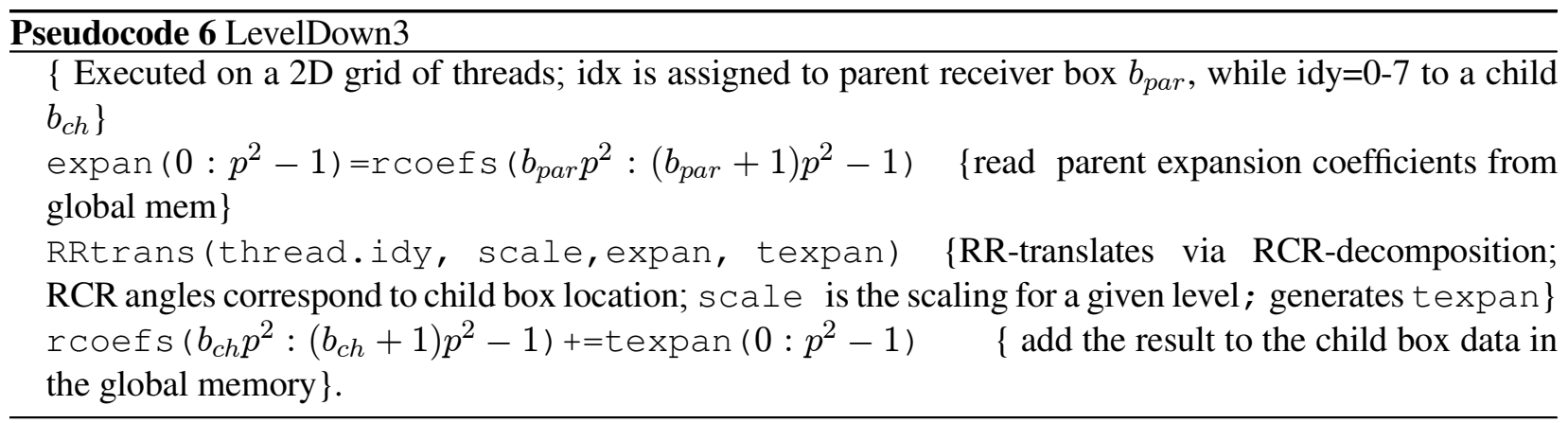

Another peculiarity of the $S \mid R$ translations is the number of input data reads needed for data corresponding to boxes in the data structure "neighborhood of the parent box at children level". Since the algorithm is adaptive, for every translation we need data on the source box and its translation parameters. Despite the fact that we are using partially ordered lists, the access pattern is rather random, which adds to the cost. Our translation data structure represents translations as a graph and each entry to the list corresponds to an edge of this graph. Based on the entry it allows retrieval of source and target indices and an encapsulated "translation index." This index can be unpacked to provide pointers to the $\alpha, \beta$, and $t$-indices of global arrays storing respective rotation and translation data. The test results are provided in the tables below.

\begin{tabular}{|l|l|l|l|l|l|l|l|l|l|}
\hline \multicolumn{9}{|l|}{ Table 6. Performance for the FMM Downward Pass } \\
\hline & $p=4$ & \multicolumn{1}{l|}{$p=8$} & & \\
\hline & & & & & \\
\hline $\max$ & CPU(s) & GPU(s) & Ratio & CPU(s) & GPU(s) & Ratio & CPU(s) & GPU(s) & Ratio \\
\hline 3 & $3.08 \mathrm{E}-02$ & $1.46 \mathrm{E}-02$ & 2.1 & $9.28 \mathrm{E}-02$ & $7.71 \mathrm{E}-02$ & 1.2 & $2.18 \mathrm{E}-01$ & $2.33 \mathrm{E}-01$ & 0.9 \\
\hline 4 & $2.03 \mathrm{E}-01$ & $6.14 \mathrm{E}-02$ & 3.3 & $9.36 \mathrm{E}-01$ & $2.65 \mathrm{E}-01$ & 3.5 & $2.39 \mathrm{E}+00$ & $7.18 \mathrm{E}-01$ & 3.3 \\
\hline 5 & $1.86 \mathrm{E}+00$ & $3.59 \mathrm{E}-01$ & 5.2 & $8.55 \mathrm{E}+00$ & $1.80 \mathrm{E}+00$ & 4.8 & $2.19 \mathrm{E}+01$ & $4.84 \mathrm{E}+00$ & 4.5 \\
\hline
\end{tabular}

Table 6 similarly to Table 4 shows relatively low accelerations of this part of the FMM. This scheme is efficient on CPU, but maybe not the best for GPU, where the cost of one random access to global memory is equal up to 150 float operations, and instead of reading precomputed data GPU may rather compute them at higher rate. Moreover for low $l_{\max }$ the GPU subroutine may even run slower than the serial CPU (!). However even this case we do not advise to switch between the CPU and GPU, since such a switch involves the slowest memory copying process (CPU-GPU), and if possible all data should stay on the GPU global memory. Performance improves as the size of the problem and, respectively, the maximum level of the octree increases and for $l_{\max }=5$ the time ratio can reach 5 or so. It is clear that even though such accelerations are not as impressive as for other subroutines, they show that it is preferable to run even the most expensive (in terms of operations with complex enough data structure, and extensive random access to the global memory) part of the FMM on the GPU rather than on the CPU. The speedup ratios look much better, if we plot the effective accelerations for the Downward Pass. Reduction of the $l_{\max }$ is very beneficial for this part of the algorithm, and the effective accelerations for problems of size $N \sim 2^{18}-2^{20}$ are in the range 30 (we have one anomalous case of 250 times speedup). Effective accelerations of order 30 are more or less consistent with the effective accelerations for the other steps of the algorithm, particularly with that for the sparse matrix vector multiplier (see Table 2). As this applies to all FMM steps this allows us to expect that the entire FMM can be speeded up 30 times or so. 


Table 7. Effective Performance (the best settings for CPU and GPU) for the FMM Downward Pass
\begin{tabular}{|l|l|l|l|l|l|l|l|l|l|l|}
\hline$l_{\max }$ & $p=4$ & $p=8$ & & & \\
\hline CPU & GPU & CPU(s) & GPU(s) & Ratio & CPU(s) & GPU(s) & Ratio & CPU(s) & GPU(s) & Ratio \\
\hline 3 & 2 & $3.08 \mathrm{E}-02$ & $4.83 \mathrm{E}-03$ & 6 & $9.28 \mathrm{E}-02$ & $2.31 \mathrm{E}-02$ & 4 & $2.18 \mathrm{E}-01$ & $6.22 \mathrm{E}-02$ & 4 \\
\hline 4 & 3 & $2.03 \mathrm{E}-01$ & $1.46 \mathrm{E}-02$ & 14 & $9.36 \mathrm{E}-01$ & $7.71 \mathrm{E}-02$ & 12 & $2.39 \mathrm{E}+00$ & $2.33 \mathrm{E}-01$ & 10 \\
\hline 5 & 4 & $1.86 \mathrm{E}+00$ & $6.14 \mathrm{E}-02$ & 30 & $8.55 \mathrm{E}+00$ & $2.65 \mathrm{E}-01$ & 32 & $2.19 \mathrm{E}+01$ & $7.18 \mathrm{E}-01$ & 30 \\
\hline 6 & 4 & $1.54 \mathrm{E}+01$ & $6.14 \mathrm{E}-02$ & 250 & N/A & N/A & N/A & N/A & N/A & N/A \\
\hline
\end{tabular}

\subsection{Overall performance}

To demonstrate a typical profile of the execution of the FMM we present Table 8, which shows the time taken by for different components of the for performing the for problem (1). Optimal settings for the CPU and GPU are used for fixed truncation numbers. The error here is measured as

$$
\epsilon_{2}=\frac{\epsilon_{2}^{(a b s)}}{\left\|\phi_{\text {exact }}(\mathbf{y})\right\|_{2}}
$$

where

$$
\epsilon_{2}^{(a b s)}=\left[\frac{1}{M} \sum_{j=1}^{M}\left|\phi_{\text {exact }}\left(\mathbf{y}_{j}\right)-\phi_{\text {approx }}\left(\mathbf{y}_{j}\right)\right|^{2}\right]^{1 / 2}, \quad\left\|\phi_{\text {exact }}(\mathbf{y})\right\|_{2}=\left[\frac{1}{M} \sum_{j=1}^{M}\left|\phi_{\text {exact }}\left(\mathbf{y}_{j}\right)\right|^{2}\right]^{1 / 2},
$$

where $\phi_{\text {exact }}(\mathbf{r})$ and $\phi_{\text {approx }}(\mathbf{r})$ are the exact (computed directly on CPU with double precision) and approximate (FMM and/or use of GPU) solutions of the problem. As shown in our report [13] $\epsilon_{2}$ is a statistically stable measure of the error (for $M \geqslant 100$ it almost does not depend on $M$ for a fixed distribution of $N$ sources). Concerning the errors, we can see that the GPU code for the cases displayed showed the error $\epsilon_{2}$ which does not exceed that for the CPU code. This error was even slightly smaller for larger $p$, which can be explained by the fact that the GPU code was executed with a smaller octree depth than on the CPU. This was beneficial not only for a good overall timing, but for the error, since more source-receiver interaction were taken into account with higher (machine) precision as they moved from the approximate dense matrix vector product to "exact" sparse matrix vector product.

\begin{tabular}{|l|l|l|l|l|l|l|}
\hline Table 8. Comparison of optimal CPU and GPU FMM: $N=1,048,576$. \\
\hline & $\mathrm{S}$ exp & Up trans & Down trans & R eval & Sparse & Total \\
\hline$p=4, \mathrm{CPU}: l_{\max }=6, \epsilon_{2}=2.3 \times 10^{-4}, \mathrm{GPU}: l_{\max }=4, \epsilon_{2}=2.3 \times 10^{-4}$ \\
\hline Serial CPU(s) & 0.34 & 0.19 & 15.06 & 0.34 & 6.31 & 22.25 \\
\hline GPU(s) & 0.011 & 0.00046 & 0.061 & 0.011 & 0.60 & 0.6835 \\
\hline Speedup (times) & 31 & 413 & 247 & 31 & 11 & 32.6 \\
\hline$p=8$, CPU: $l_{\max }=5, \epsilon_{2}=8.8 \times 10^{-6}, \mathrm{GPU}: l_{\max }=4, \epsilon_{2}=8.3 \times 10^{-6}$. \\
\hline Serial CPU(s) & 0.83 & 0.12 & 8.42 & 0.85 & 40.95 & 51.17 \\
\hline GPU(s) & 0.020 & 0.00218 & 0.265 & 0.021 & 0.60 & 0.9082 \\
\hline Speedup (times) & 42 & 55 & 32 & 40 & 68 & 56.3 \\
\hline$p=12, \mathrm{CPU}: l_{\max }=5, \epsilon_{2}=1.3 \times 10^{-6}, \mathrm{GPU}: l_{\max }=4, \epsilon_{2}=9.5 \times 10^{-7}$ \\
\hline Serial CPU(s) & 1.91 & 0.33 & 21.30 & 1.93 & 40.95 & 66.56 \\
\hline GPU(s) & 0.040 & 0.00562 & 0.72 & 0.030 & 0.59 & 1.395 \\
\hline Speedup (times) & 48 & 59 & 30 & 67 & 69 & 47.7 \\
\hline
\end{tabular}

This table demonstrates overall speedups of the entire FMM run in the range 30-60 for the million size case, which is consistent with the efficient speedups for all components. It is well seen from data for $p=8$ 


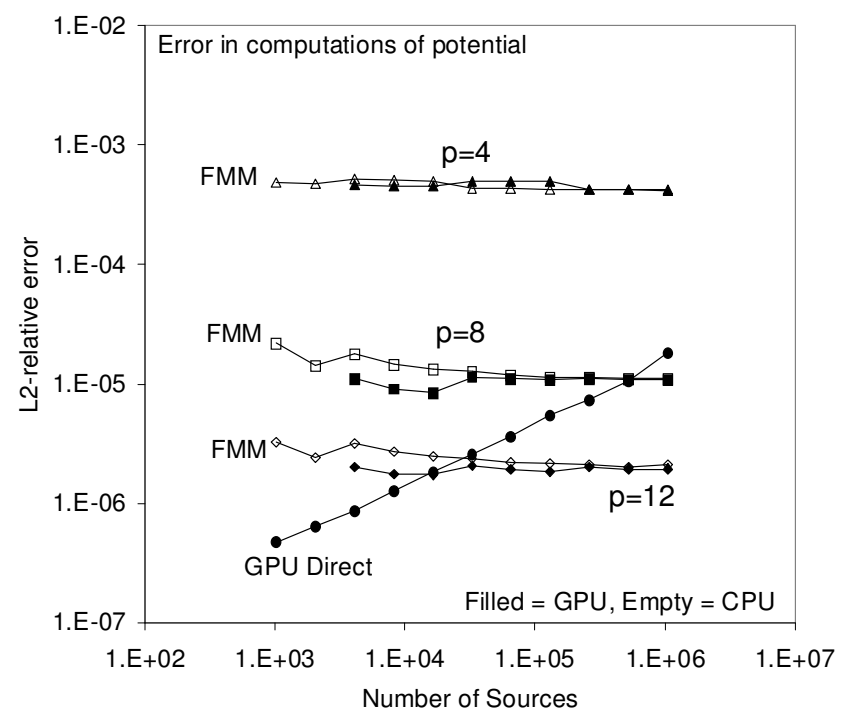

Figure 10: $\epsilon_{2}$ error (see (36)) for evaluation of sum (1) using the FMM on CPU (filled markers) and on GPU (empty markers). Also the error for direct summation on the GPU is shown. The errors are obtained by comparisons with the double precision direct computations on CPU for a grid of $N$ sources of intensity 1 and 729 receivers.

and $p=12$. The case $p=4$ shows higher imbalance of component accelerations, which is due to the larger difference in optimal $l_{\max }$ for the CPU and GPU realizations.

It may be noticed that for all cases considered the sparse matrix multiplier takes a substantial part of the total execution time. That puts a lighter weight on optimization of the translation subroutines. Indeed, assuming that the entire dense matrix vector product (the first four components in Table 8) are executed with zero time, the total time will change at most by a factor of 2.3 for $p=12$ and only by a factor of 1.14 for $p=4$. Since the sparse matrix vector is optimized, even for the best possible translation methods we cannot expect an increase of the overall performance by orders of magnitude, and at most by a factor of approximately 2. This is totally different from the model of the FMM on CPU, where efficiency of the translation has a heavy impact on total time as it controls the depth of the octree, and one can therefore expect orders of magnitude accelerations by balancing the costs of the dense and sparse parts of the FMM.

\subsubsection{FMM and roundoff errors}

There are two types of errors associated with the use of the FMM. First, the errors due to truncation of infinite series, which are controlled by specification of the truncation number $p$, and, second, the errors due to roundoff in various operations and intrinsic functions. To study the errors we created a benchmark case, where all sources have intensity $q=1$ and distributed in a regular grid over the unit cube. 729 evaluation points were also put in a grid covering the domain. The error measure used is $\epsilon_{2}$ in Eq. (36). The "exact" solution was computed on the CPU using double and quadruple-precision and direct summation (double precision and quadruple-precision provided consistent results that are far more accurate than the single precision computations). Single precision results obtained on the CPU and GPU using the FMM and direct summation on GPU were compared with the "exact" solution. The use of grids and constant source intensity for error check was dictated by the desire to eliminate any possible error in input (positions and intensities). The results are shown in Fig. 10. 
The CPU and GPU implementations of the FMM produce approximately the same results, which clearly show that the error is controlled by $p$ for the cases studied. A small difference in the FMM errors is explained by the fact that lower $l_{\max }$ is used for the GPU. Second, it is seen that the direct sums on the GPU are computed with error which increases with $N$. We relate this to accumulation of roundoff errors in direct summation when computing large sums.

On the other hand, for large sums the FMM demonstrates more stability to roundoff errors. Indeed, only a limited number of source-receiver interactions are taken into account directly (sparse matrix) and computations of the far field via expansions involve very limited number of operations (limited number of expansion terms and a few stable translation operators). So if we speak about summation with machine precision (i.e. precision available through the use of standard hardware on the GPU) it appears that the use of the FMM with moderate truncation numbers ( $p=8,12$ in our case) is sufficient to reach this level of accuracy. Moreover, the use of the FMM may be even preferable (Fig. 10 shows that FMM on GPU is more accurate than direct summation for $N \gtrsim 50,000$ and $p=12$ and for $N \gtrsim 1,000,000$ and $p=8$ ). We also performed more error tests with random distributions, different number of evaluation points, and random source intensities, which confirm this finding.

\subsubsection{FMM time}

Finally we present the performance (timing) results for different truncation numbers. All results in this section are obtained for computations of both the potential and its gradient as given by Eqs (1) and (2). Tests were performed on our benchmark problem ( $N$ random sources of random intensities $q \in(0,1)$, and independent set of $M=N+1$ random receivers). Table 11 shows some numbers obtained for $N=2^{20}$. Note that these numbers are somehow random, as we used random realizations, while we did each computation at least 10 times to be sure that the results are stable. One also can compare these data with that given in Table 10 to see the cost of computations of gradient.

\begin{tabular}{|c|c|c|c|c|c|c|c|c|}
\hline \multicolumn{3}{|l|}{$p=4$} & \multicolumn{3}{|l|}{$p=8$} & \multicolumn{3}{|l|}{$p=12$} \\
\hline CPU(s) & GPU(s) & $\begin{array}{l}\text { Ratio } \\
\end{array}$ & CPU(s) & GPU(s) & Ratio & CPU(s) & GPU(s) & Ratio \\
\hline 28.37 & 0.979 & 29 & 88.09 & 1.227 & 72 & 116.1 & 1.761 & 66 \\
\hline
\end{tabular}

Fig. 11 shows wall clock time required to run the FMM and direct summation on a single CPU and GPU. We note a huge acceleration for direct summation, which should be reduced if one uses more proper CPU memory management, SSE instructions, and high performance libraries (we compared with a naive straightforward implementation, when the direct sum routine simply executes a memory aligned nested loop).

For $p=4$ at large enough $N$ both the CPU and GPU times depend on $N$ linearly, which is consistent with the theory of the FMM. It interesting also to note that the so-called break-even point, i.e. the point at which the time of the direct summation and the FMM is equal for the FMM running on the GPU is somewhere around $N \sim 10^{4}$, while for the FMM on CPU this point is below $N=10^{3}$. So the use of the FMM is efficient on the GPU for larger $N$. It is also interesting to note the second break-even point, where the direct sum evaluator for the GPU crosses the dependence for serial CPU. This point is located $N \sim 2 \times 10^{5}$, which also shows that it is maybe more efficient (do not forget about the error!) to compute problems with smaller $N$ on the GPU directly than use rather complex FMM strategy on the CPU. In any way due to linear scaling any FMM at some $N$ beats any direct method scaled quadratically and for million size problems the FMM is more efficient method. We also note that it is possible to compute even million size problem using the direct method on GPU, which takes a reasonable 100 s time. But the FMM on GPU performs this computations for times less than for $1 \mathrm{~s}(0.98 \mathrm{~s})$. It is accepted by several researchers in the N-body community (e.g. [25]) that the number of float operations for a single potential+gradient evaluation is 38 , and this is the figure used 


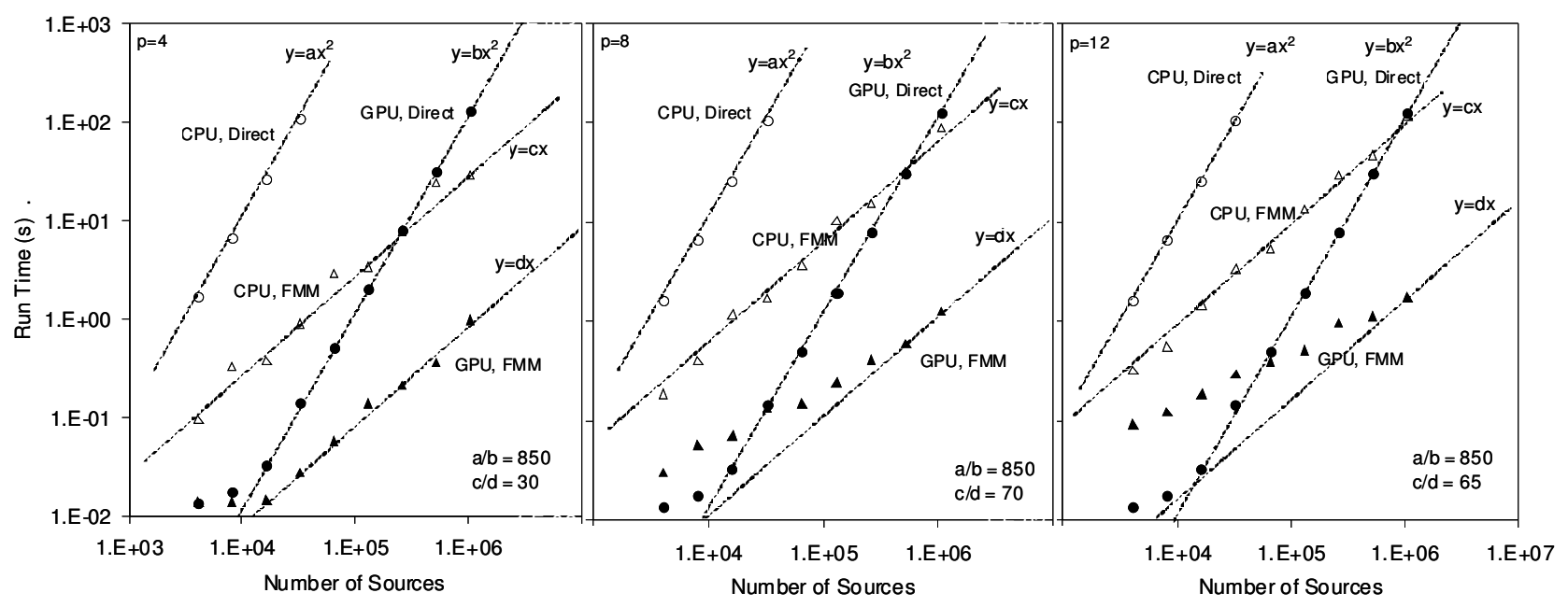

Figure 11: FMM wall clock time (in seconds) for serial CPU code (one core of $2.67 \mathrm{GHz}$ Intel Core 2 extreme QX is employed) and for GPU (NVIDIA GeForce 8800 GTX) for different truncation numbers $p$ (potential+gradient). Also direct summation timing is displayed for both architectures. No SSE optimizations for the CPU were used.

for comparisons in e.g., [16]. In this case the effective rate due to algorithmic, FMM, and, hardware, GPU, acceleration for the $N=2^{20}$ case reaches number about 43 Tflops (!).

Case $p=8$ has some similarities and differences with the case $p=4$. The similarity is in fact that the FMM is greatly accelerated by the use of the GPU. The difference is that the time dependence of the FMM on GPU on $N$ seems not linear. Since $p$ influences only the dense matrix vector product this is manifestation of the fact that our approach based on parallelization of operations on boxes does not reach asymptotic saturation in the range of $N$, for which the current study is conducted. Indeed for large $p$ the downward pass costs as much as as the sparse matrix vector multiplication, while at levels $l_{\max }=3$ and 4 it still does not reach its asymptotic dependence on $l_{\max }$, or $N$. However our tests for larger $l_{\max }$, which we did not present in Table 8 show that, in fact such saturation occurs and accelerations for the CPU analog running at the same level stabilize around 5. So it is anticipated that at larger $N$ the dependence of the GPU time on $N$ will be linear if we keep $s_{\max }$ to be a constant $\left(s_{\max }=320\right.$ ). We also can note that the break even point for the CPU is still below $N=10^{3}$ while this point for the GPU shifts towards larger $N \sim 3 \times 10^{4}$. Our estimates show that the effective rate of computations in this case 34 Tflops for $N=2^{20}$.

Case $p=12$ is qualitatively similar to case $p=8$ and the same explanation for the nonlinear behavior of the FMM run time on the GPU applies to it. The dependence of the run time on $l_{\max }$ rather than on $N$ is expressed here even more than in the graph for $p=8$. The break-even point shifts further towards to larger $N$ and is around $N \sim 7 \times 10^{4}$ and the effective rate in this case is about 24 Tflops for $N=2^{20}$.

\section{Conclusions}

We have accelerated the run time of the FMM algorithm on the GPU in the range 30-70 (depending on the accuracy) which is equivalent to the computing rates in the 24-43 Teraflop equivalent rates [25] on a single GPU and showed that it is feasible to program a complicated algorithm such as the FMM on the GPU. This should permit the use of these architectures in several scientific computation problems where the FMM or hierarchical algorithms can be profitably used. Our analysis showed the following conclusions: 
Optimal cluster size on a GPU: On the CPU the local direct sum cost is proportional to the cluster size, while the cost of the far-field evaluation decreases with increasing cluster size. Accordingly, it is possible to find an optimal setting. In contrast, on the GPU architecture access to global memory is expensive. As there are more boxes, and more clusters, for each cluster we need to perform random access to the global memory for its neighbor list. Because of this, there is an optimal size for the cluster (independent of the FMM far field evaluation). For optimality, the cluster size should be larger than certain value determined by the hardware. This optimal size is larger than that on the CPU, and, correspondingly, the FMM is faster than the direct product on the GPU only for larger problem sizes. The FMM should be run on the architectures at these optimal settings. The increase of the cluster size on the GPU also results in more effective acceleration of all other components of the FMM and improves the accuracy of the computations, since a larger fraction of points are computed directly.

Parallelization Strategies for the FMM: Each of the FMM operations require differing amounts of local memory. For example, each translation requires the use of a relatively high amount of constant translation data, which is difficult to fit into the small amount of constant memory available, and more complex parallelization is needed. Accordingly we used two different parallelization strategies of TpB ("thread per box") and BpB ("block (of threads) per box"). The upward pass (which requires creating multipole expansion coefficients, and multipole-to-multipole translation could be done via the TpB strategy, while in the downward pass, the multipole-to-local translation and local summation required a $\mathrm{BpB}$ strategy, while the local expansion evaluation could be done via a TpB strategy.

Error in the Computed Solution: Current GPUs are single precision architectures, and the behavior of the error in the solution is of interest. As a general conclusion, we can say that the GPU behaves as expected. An interesting feature of the GPU sum is that at some point the accumulated arithmetic errors due to computing large sums of (reciprocal) square roots becomes much larger, and the FMM becomes more accurate than direct summation.

\section{Acknowledgements}

We would like to acknowledge funding of this work via award NNX07CA22P from NASA to Fantalgo, LLC. In addition NG was also partially supported by the Center for Multiscale Plasma Dynamics (CMPD), a Department of Energy Fusion Science Center, during the writing of this paper.

\section{References}

[1] NVIDIA CUDA Compute Unified Device Architecture Programming Guide, V. 1.0, 06/01/2007. http://developer.download.nvidia.com/compute/cuda/1_0/NVIDIA_CUDA_Programming_Guide_1.0.pdf)

[2] M. Abramowitz, and I. A. Stegun. Handbook of Mathematical Functions. Dover Publications, 1965.

[3] D.H. Bailey. Multiprecision translation and execution of Fortran programs. ACM Trans. on Mathematical Software, 19(3):288-319, 1993.

[4] H. Cheng, L. Greengard, and V. Rokhlin. A fast adaptive multipole algorithm in three dimensions. J. Comput. Phys., 155, 1999, 468-498.

[5] J.J. Dongarra and F. Sullivan. The top 10 algorithms. Computing in Science \& Engineering, 2, 2000, 22-23.

[6] M.A. Epton and B. Dembart. Multipole translation theory for the three-dimensional Laplace and Helmholtz equations. SIAM J. Sci. Comput., 16(4), 1995, 865-897. 
[7] D. Goeddeke, R. Strzodka, and S. Turek. Accelerating double precision FEM simulations with GPUs. Frontiers in Simulation (Proceedings of ASIM 2005), Edited by F. Hülsemann, M. Kowarschik, and U. Rüde, SCS Publishing House, 2005, 139-144.

[8] L. Greengard and V. Rokhlin. A fast algorithm for particle simulations. J. Comput. Phys., 73, 1987, 325-348.

[9] L. Greengard and W.D. Gropp. A parallel version of the fast multipole method. Comput. Math. App. 20, 1990, 63-71.

[10] L. Greengard and V. Rokhlin. A new version of the fast multipole method for the Laplace equation in three dimensions. Acta Numerica, 6, 1997, 229-269.

[11] N.A. Gumerov and R. Duraiswami. Fast Multipole Methods for the Helmholtz Equation in Three Dimensions. Elsevier, Oxford, UK, 2005.

[12] N.A. Gumerov and R. Duraiswami. Fast multipole method for the biharmonic equation in three dimensions. J. Comput. Physics, 215 (1), 2006, 363-383.

[13] N.A. Gumerov and R. Duraiswami. Comparison of the efficiency of translation operators used in the fast multipole method for the 3D Laplace equation. University of Maryland Technical Report UMIACSTR-\#2003-28, (http://www.cs.umd.edu/Library/TRs/CS-TR-4701/CS-TR-4701.pdf).

[14] N.A. Gumerov, R. Duraiswami, and W. Dorland. Middleware for programming NVIDIA GPUs from Fortran 9X. (poster presentation at Supercomputing 207: online at www.umiacs.umd.edu/ ramani/pubs/Gumerov_SC07_poster.pdf)

[15] T. Fukushige, J. Makino, and A. Kawai. GRAPE-6A: A single-card GRAPE-6 for parallel PC-GRAPE cluster systems. PASJ: Publ. Astron. Soc. Japan 57, 2005, 1009-1021.

[16] T. Hamada and T. Iitaka. The Chamomile scheme: an optimized algorithm for N-body simulations on programmable graphics processing units (posted on arXiv: astro-ph/0703100v1, 6 Mar 2007).

[17] J. Makino, T. Fukushige, M. Koga, and K. Namura. GRAPE-6: Massively-parallel special-purpose computer for astrophysical particle simulations. Publication of Astronomical Society of Japan, 55, 2001, 1163-1187.

[18] L. Nyland, M. Harris, and J. Prins. Fast N-body Simulations with CUDA. in Hubert Nguyen (ed.), GPU Gems 3, 31, 677-695. Addison Wesley, August 2007.

[19] J.P. Singh, C. Holt, J.L. Hennessy, and A. Gupta. A parallel adaptive fast multipole method. in: Proceedings of the 1993 ACM/IEEE Conference on Supercomputing, December 1993.

[20] J.P. Singh, J.L. Hennessy, and A. Gupta. Implications of hierarchical N-body methods for multiprocessor architectures, ACM Trans. Comput. Systems 13 (2), 1995, 141-202.

[21] J.P. Singh, C. Holt, T. Totsuka, A. Gupta, and J.L. Hennessy. Load balancing and data locality in adaptive hierarchical N-body methods: B-H, FMM, and radiosity. J. Parallel Distrib. Comput. 27, 1995, 118-141.

[22] J.E. Stone, J.C. Phillips, P.L. Freddolino, D.J. Hardy, L.G. Trabuco, and K. Schulten. Accelerating molecular modeling applications with graphics processors, J. Comput. Chem., in press, 2007. 
[23] John D. Owens, David Luebke, Naga Govindaraju, Mark Harris, Jens Krüger, Aaron E. Lefohn, and Timothy J. Purcell. A survey of general-purpose computation on graphics hardware. Computer Graphics Forum, 26:80-113, 2007.

[24] S.-H. Teng. Provably good partitioning and load balancing algorithms for parallel adaptive N-body simulation. SIAM J. Sci. Comput. 19(2), 1998, 635-656.

[25] S. Warren, M, K. Salmon, J, J. Becker, D, P. Goda, M, and T. Sterling. Pentium Pro inside: I. A treecode at 430 Gigaflops on ASCI Red, II. Price/Performance of \$50/Mflop on Loki and Hyglac," in Proc. Supercomputing 97, in CD-ROM. IEEE, Los Alamitos, CA, 1997.

[26] C.A. White and M. Head-Gordon. Rotation around the quartic angular momentum barrier in fast multipole method calculations. J. Chem. Phys., 105(12), 1996, 5061-5067.

[27] M. J. Stock and A. Gharakhani. Toward efficient GPU-accelerated N-body simulations, in 46th AIAA Aerospace Sciences Meeting and Exhibit, AIAA 2008-608, January 2008, Reno, Nevada.

[28] P. Zwart, F. Simon, R.G. Belleman, P.M. Geldof. High-performance direct gravitational N-body simulations on graphics processing units. New Astronomy, 12(8), 2007, 641-650. 